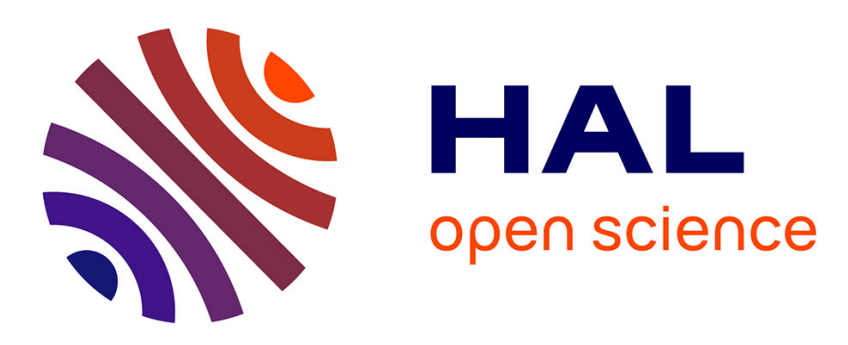

\title{
Solving multizone and multicrack elastostatic problems: a fast multipole symmetric Galerkin boundary element method approach
}

Quoc Tuan Trinh, Saida Mouhoubi, Cyrille Chazallon, Marc Bonnet

\section{- To cite this version:}

Quoc Tuan Trinh, Saida Mouhoubi, Cyrille Chazallon, Marc Bonnet. Solving multizone and multicrack elastostatic problems: a fast multipole symmetric Galerkin boundary element method approach. Engineering Analysis with Boundary Elements, 2015, 50, pp.486-495. 10.1016/j.enganabound.2014.10.004 . hal-01082068

\section{HAL Id: hal-01082068 https://hal.science/hal-01082068}

Submitted on 12 Nov 2014

HAL is a multi-disciplinary open access archive for the deposit and dissemination of scientific research documents, whether they are published or not. The documents may come from teaching and research institutions in France or abroad, or from public or private research centers.
L'archive ouverte pluridisciplinaire HAL, est destinée au dépôt et à la diffusion de documents scientifiques de niveau recherche, publiés ou non, émanant des établissements d'enseignement et de recherche français ou étrangers, des laboratoires publics ou privés. 


\title{
Solving multizone and multicrack elastostatic problems: a fast multipole symmetric Galerkin boundary element method approach
}

\author{
Quoc Tuan TRINH ${ }^{\mathrm{a}}$, Saida MOUHOUBI ${ }^{\mathrm{a}}$, Cyrille CHAZALLON ${ }^{\mathrm{a}}$, Marc BONNET ${ }^{\mathrm{b}}$, \\ ${ }^{a}$ ICUBE (UMR 7357 CNRS), INSA de Strasbourg, 24 bd de la victoire, 67084 Strasbourg Cedex, FRANCE \\ ${ }^{b}$ POems (UMR 7231 CNRS-INRIA-ENSTA), ENSTA, 828 bd des maréchaux, 91120 Palaiseau, FRANCE
}

\begin{abstract}
Symmetric Galerkin boundary element methods (SGBEMs) for three-dimensional elastostatic problems give rise to fully-populated (albeit symmetric) matrix equations, entailing high solution times for large models. This article is concerned with the formulation and implementation of a multi-level fast multipole SGBEM (FM-SGBEM) for multi-zone elasticity problems with cracks. The subdomain coupling approach is based on a minimal set of interfacial unknowns (i.e. one displacement and one traction vector at any interfacial point) that are defined globally for the complete multizone configuration. Then, unknowns for each subdomain are defined in terms of the global unknowns, with appropriate sign conventions for tractions induced by subdomain numbering. This formulation (i) automatically enforces the perfect-bonding transmission conditions between subdomains, and (ii) is globally symmetric. The subsequent FM-SGBEM basically proceeds by assembling contributions from each subregion, which can be computed by means of an existing single-domain FM-SGBEM implementation such as that previously presented by the authors (EABE, 36:1838-1847, 2012). Along the way, the computational performance of the FM-SGBEM is enhanced through (a) suitable storage of the near-field contribution to the SGBEM matrix equation and (b) preconditioning by means of nested GMRES. The formulation is validated on numerical experiments for 3D configurations involving many cracks and inclusions, and of sizes up to $N \approx 10^{6}$.
\end{abstract}

Keywords: fast multipole method (FMM), generalized minimal residuals (GRMES), symmetric Galerkin boundary element method (SGBEM), fracture mechanics, multizone.

\section{Introduction}

Boundary integral equation (BIE) formulations $[3,40]$ arise naturally in problems involving piecewise-homogeneous materials (hereinafter referred to as multi-zone problems) or cracks. They are at the root of many analytical or semi-analytical solutions for canonical problems, e.g. ellipsoidal inhomogeneities [15, 33] or penny-shaped cracks [42]. Moreover, their numerical implementation by means of boundary elements permit accurate solutions for e.g. stress concentrations [25] or stress intensity factors, while greatly simplifying the meshing of complex configurations (e.g. numerical homogenization problems involving many inclusions embedded in an elastic matrix [30]).

Elasticity problems for multi-zone cracked configurations are involved in the modelling and analysis of e.g. composite materials or geomechanical systems with fractures. Traction BIEs naturally occur in connection with both multi-zone configurations (as transmission conditions involve tractions) and cracks (because of the well-known degeneracy of displacement-based BIEs in that case). Collocation-based formulations, while feasible, are then somewhat cumbersome as they require $C^{1, \alpha}$ regularity of the density at the collocation points and specially designed singular integration procedures. In contrast, the symmetric Galerkin form of traction BIEs (SGBIE) only requires $H^{1 / 2}$ densities for the hypersingular integral operator, making $C^{0}$ boundary element interpolations suitable (see [6] for an early implementation of SGBIEs for planar cracks, and also [20, 21, 17, 4, 29], Preprint submitted to Eng. Anal. Bound. Elem.

October 22, 2014 
among many references on this kind of treatment). This, together with symmetry and underlying energy-based variational principles, makes SGBIE formulations a natural approach for problems involving multi-zone configurations and cracks. For example, a fully symmetric BIE treatment of multi-zone problems is proposed for heat transfer problems in [21], see also [26] (energy-based variational $\mathrm{BIE}$ for media with piecewise-constant conductivity), [18, 31] (elastostatics), [9] (2D piecewise-homogeneous configurations with cohesive cracks).

The symmetric Galerkin BEM (SGBEM) yields symmetric but dense matrices, see e.g. the references in the survey article [5]. As with other forms of BEM, this makes standard implementations impractical for large-scale problems because of excessive (respectively $O\left(N^{2}\right), O\left(N^{2}\right)$ and $O\left(N^{3}\right)$ ) complexities for set-up, storage and direct linear solvers ( $N$ denoting the number of BEM unknowns), triggering a recourse to acceleration strategies. Most of these entail using iterative solvers while taking advantage of the blockwise approximate low-rank character of integral operators (at least for blocks encoding influences between spatially well-separated parts of the featured boundary). Acceleration approaches include the fast multipole method (FMM) [23], now available for many engineering problems (e.g. Stokes flows [19, 16], acoustics [1, 46], electromagnetism [10], elastodynamics [45, 7]). Regarding crack problems, fast multipole BEMs (FM-BEMs) have been investigated in e.g. [48, 47, 28] for 3D unbounded bodies. FM-SGBEMs have also been developed in several contexts (see e.g. [35] for potential problems, [44] for electromagnetic problems or [28] for cracks in unbounded media). Moreover, other acceleration approaches based on e.g. hierarchical matrices also experience rapid development, see e.g. [24, 2]. For static (Laplace-like) problems, the multi-level form of the FMM achieves solution times of order $O(N)$ per linear solver iteration.

This article is concerned with the formulation and implementation of a multi-level fast multipole SGBEM (FM-SGBEM) for multi-zone elasticity problems with cracks, and is intended as a continuation of [36] where FM-SGBEM were investigated for homogeneous elastic solids containing (possibly many) cracks. The subdomain coupling approach, following [21, 18], is based on a minimal set of interfacial unknowns (i.e. one displacement and one traction vector at any interfacial point) that are defined globally for the complete multizone configuration. Then, unknowns for each subdomain are defined in terms of the global unknowns, with appropriate sign conventions for tractions that depend on subdomain numbering. This formulation (i) automatically enforces the perfect-bonding transmission conditions between subdomains, (ii) uses a minimal set of interfacial unknowns (e.g. without recourse to Lagrange multipliers) and (iii) is globally symmetric. The subsequent FM-SGBEM basically proceeds by summation of contributions from each subregion, which can be computed by means of an existing single-domain FM-SGBEM implementation.

The article is organised as follows. The FM-SGBEM formulation for single-region problems with cracks is summarized in Sec. 2. The enhancement of its computational performance through (a) suitable storage of the near-field contribution to the SGBEM linear system and (b) preconditioning by means of nested GMRES is then examined in Sec. 3, and tested on multiple-crack configurations of size up to $N \approx 3 \times 10^{6}$. Next, the proposed SGBIE treatment of 3D multi-zone problems with cracks and the resulting FM-SGBEM are presented in Sec. 4. Numerical experiments on configurations involving many cracks and inclusions, and size up to $N \approx 10^{6}$, are reported in Sec. 5 .

\section{Mathematical background}

Letting $\boldsymbol{x}, \tilde{\boldsymbol{x}} \in \mathbb{R}^{3}$ denote two generic points in the three-dimensional physical space, the wellknown Kelvin (elastostatic) fundamental displacement and traction tensors are given by

$$
\boldsymbol{U}(\boldsymbol{x}, \tilde{\boldsymbol{x}})=\frac{1}{16 \pi \mu(1-\nu) r}[(3-4 \nu) \hat{\boldsymbol{r}} \otimes \hat{\boldsymbol{r}}+\boldsymbol{I}]
$$




$$
\boldsymbol{T}(\boldsymbol{x}, \tilde{\boldsymbol{x}})=-\frac{1}{8 \pi(1-\nu) r^{2}}[(3 \hat{\boldsymbol{r}} \otimes \hat{\boldsymbol{r}}+(1-2 \nu) \boldsymbol{I}) \hat{\boldsymbol{r}} \cdot \boldsymbol{n}(\boldsymbol{x})+(1-2 \nu)(\boldsymbol{n}(\boldsymbol{x}) \otimes \hat{\boldsymbol{r}}+\hat{\boldsymbol{r}} \otimes \boldsymbol{n}(\boldsymbol{x}))],
$$

where $\mu, \nu$ are the shear modulus and Poisson's ratio characterizing the isotropic elastic constitutive material, and having set

$$
\boldsymbol{r}=\boldsymbol{x}-\tilde{\boldsymbol{x}}, \quad r=\|\boldsymbol{r}\|, \quad \hat{\boldsymbol{r}}=\boldsymbol{r} / r
$$

Then, the elastostatic single-layer potential $\mathcal{V}$ and double-layer potential $\mathcal{W}$ are defined [27], for a generic piecewise-smooth surface $S$ and vector-valued densities $\boldsymbol{u}, \boldsymbol{p}: S \mapsto \mathbb{R}^{3}$, by

$$
\mathcal{V}[S, \boldsymbol{p}](\tilde{\boldsymbol{x}})=\int_{S} \boldsymbol{U}(\boldsymbol{x}, \tilde{\boldsymbol{x}}) \cdot \boldsymbol{p}(\boldsymbol{x}) \mathrm{d} S_{x}, \quad \mathcal{W}[S, \boldsymbol{u}](\tilde{\boldsymbol{x}})=\int_{S} \boldsymbol{T}(\boldsymbol{x}, \tilde{\boldsymbol{x}}) \cdot \boldsymbol{u}(\boldsymbol{x}) \mathrm{d} S_{x} \quad\left(\tilde{\boldsymbol{x}} \in \mathbb{R}^{3} \backslash S\right)
$$

The symmetric Galerkin boundary integral equation (SGBIE) formulation for a cracked solid can then be formulated in terms of the boundary traces of the potentials (2) and their derivatives with respect to $\tilde{\boldsymbol{x}}$. Moreover, the notational conventions adopted in (2) will facilitate writing the SGBIE formulation for multi-domain configurations.

\subsection{Symmetric Galerkin BIE single-region formulation}

Consider for now a homogeneous elastic solid $\Omega$ containing a traction-free crack (or set thereof), see Fig. 1. The external boundary of $\Omega$ is denoted by $S$, while the (possibly non-connected) open surface $S_{c}$ is the geometrical locus of the (set of) internal crack(s). Only internal cracks are considered in this work, i.e. the closure of $S_{c}$ is assumed to have no intersection with $S$. The displacement discontinuity $\phi$ on $S_{c}$, also termed crack opening displacement (COD), is defined by $\phi(\boldsymbol{x}):=\boldsymbol{u}\left(\boldsymbol{x}^{+}\right)-\boldsymbol{u}\left(\boldsymbol{x}^{-}\right)$(with $\boldsymbol{x}^{+} \in S_{c}^{+}$and $\boldsymbol{x}^{-} \in S_{c}^{-}, S_{c}^{+}$and $S_{c}^{-}$being the upper and lower crack faces, the unit normal to $S_{c}$ being conventionally directed from $S_{c}^{-}$to $S_{c}^{+}$, i.e. $\boldsymbol{n}:=\boldsymbol{n}^{-}=-\boldsymbol{n}^{+}$on $\left.S_{c}\right)$. For this configuration, the Somigliana displacement integral identity reads

$$
\boldsymbol{u}(\tilde{\boldsymbol{y}})+\mathcal{W}[S, \boldsymbol{u}](\tilde{\boldsymbol{y}})-\mathcal{W}\left[S_{c}, \boldsymbol{\phi}\right](\tilde{\boldsymbol{y}})-\mathcal{V}[S, \boldsymbol{p}](\tilde{\boldsymbol{y}})=\mathbf{0} \quad(\tilde{\boldsymbol{y}} \in \Omega)
$$

Let $\tilde{\boldsymbol{x}}$ denote a regular point of $\partial \Omega$ (i.e. such that $\partial \Omega$ is smooth at $\tilde{\boldsymbol{x}}$ ). The integral identities underlying the SGBIE formulation stem from writing the interior boundary traces (i.e. the limiting forms as $\tilde{\boldsymbol{y}} \in \Omega \rightarrow \tilde{\boldsymbol{x}} \in \partial \Omega$ ) of (i) identity (3) and (ii) the associated traction identity obtained by applying the traction operator $\boldsymbol{w} \mapsto \boldsymbol{t}[\boldsymbol{w}](\tilde{\boldsymbol{y}}):=\boldsymbol{\sigma}[\boldsymbol{w}](\tilde{\boldsymbol{y}}) \cdot \boldsymbol{n}(\tilde{\boldsymbol{x}})$ to identity (3). The elastic potentials (2) have the well-known limiting forms [27]

$$
\lim _{\tilde{\boldsymbol{y}} \in \Omega \rightarrow \tilde{\boldsymbol{x}} \in S} \mathcal{V}[S, \boldsymbol{p}](\tilde{\boldsymbol{y}})=\mathcal{V}[S, \boldsymbol{p}](\tilde{\boldsymbol{x}}), \quad \lim _{\tilde{\boldsymbol{y}} \in \Omega \rightarrow \tilde{\boldsymbol{x}} \in S} \mathcal{W}[S, \boldsymbol{u}](\tilde{\boldsymbol{y}})=-\frac{1}{2} \boldsymbol{u}(\tilde{\boldsymbol{x}})+\mathcal{K}[S, \boldsymbol{u}](\tilde{\boldsymbol{x}})
$$

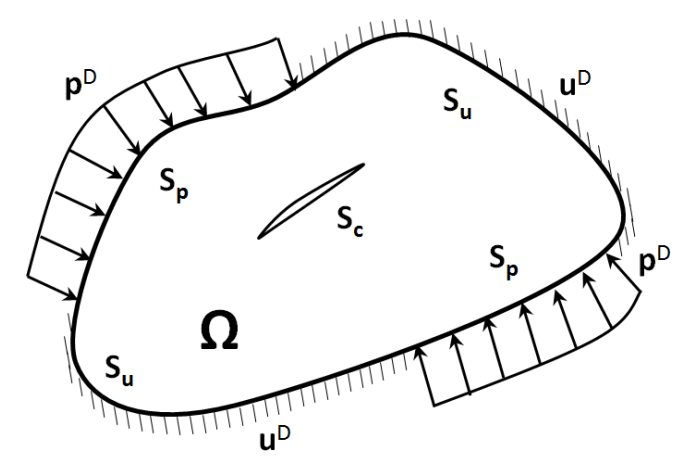

Figure 1: Solid containing a crack 
with $\mathcal{K}[S, \boldsymbol{u}](\tilde{\boldsymbol{x}})$ defined by

$$
\mathcal{K}[S, \boldsymbol{u}](\tilde{\boldsymbol{x}})=\mathrm{PV} \int_{S} \boldsymbol{T}(\boldsymbol{x}, \tilde{\boldsymbol{x}}) \cdot \boldsymbol{u}(\boldsymbol{x}) \mathrm{d} S_{x}
$$

(the symbol PV indicating that the integral is to be understood in the Cauchy principal value sense). As a result, the weak form of the interior boundary trace of (3) reads

$$
\frac{1}{2}\langle\boldsymbol{u}, \tilde{\boldsymbol{p}}\rangle_{S}+\langle\mathcal{K}[S, \boldsymbol{u}], \tilde{\boldsymbol{p}}\rangle_{S}-\left\langle\mathcal{W}\left[S_{c}, \boldsymbol{\phi}\right], \tilde{\boldsymbol{p}}\right\rangle_{S}-\langle\mathcal{V}[S, \boldsymbol{p}], \tilde{\boldsymbol{p}}\rangle_{S}=0
$$

where $\tilde{\boldsymbol{p}}$ is a test function supported on $S$ and $\langle f, g\rangle_{S}:=\int_{S} f g \mathrm{~d} S$ denotes the $L^{2}(S)$ scalar product.

Then, the interior boundary traces of the tractions associated to the potentials (2) are given by

$$
\begin{aligned}
\lim _{\tilde{\boldsymbol{y}} \in \Omega \rightarrow \tilde{\boldsymbol{x}} \in S} \boldsymbol{t}[\mathcal{V}[S, \boldsymbol{p}]](\tilde{\boldsymbol{y}}) & =-\frac{1}{2} \boldsymbol{p}(\tilde{\boldsymbol{x}})+\mathcal{K}^{\prime}[S, \boldsymbol{p}](\tilde{\boldsymbol{x}}), \\
\lim _{\tilde{\boldsymbol{y}} \in \Omega \rightarrow \tilde{\boldsymbol{x}} \in S} \boldsymbol{t}[\mathcal{W}[S, \boldsymbol{u}]](\tilde{\boldsymbol{y}}) & =\mathcal{D}[S, \boldsymbol{u}](\tilde{\boldsymbol{x}})
\end{aligned}
$$

with the integral operators $\mathcal{K}^{\prime}$ and $\mathcal{D}$ represented, for any test function $\tilde{\boldsymbol{u}}$, by $\left\langle\mathcal{K}^{\prime}[S, \boldsymbol{p}], \tilde{\boldsymbol{u}}\right\rangle_{S}=$ $\langle\mathcal{K}[S, \tilde{\boldsymbol{u}}], \boldsymbol{p}\rangle_{S}$ (i.e. $\mathcal{K}^{\prime}$ is the $L^{2}(S)$-adjoint of $\mathcal{K}$ ) and $[29]$

$$
\langle\mathcal{D}[S, \boldsymbol{u}], \tilde{\boldsymbol{u}}\rangle_{S}=\int_{S} \int_{S}[R \Delta u]_{i q}(\boldsymbol{x}) B_{i k q s}(\boldsymbol{r})[R \Delta \tilde{u}]_{k s}(\tilde{\boldsymbol{x}}) \mathrm{d} S_{\tilde{x}} \mathrm{~d} S_{x}
$$

In (7), the surface curl operator $R[34]$ is defined (in component notation) by

$$
[R u]_{k s}(\tilde{\boldsymbol{x}})=e_{j f s} n_{j} u_{k, f}(\tilde{\boldsymbol{x}})
$$

(with $e_{j f s}$ denoting the permutation symbol), while the weakly singular kernel $B_{i k q s}$ is given by [29]

$$
B_{i k q s}(\boldsymbol{r})=\frac{1}{8 \pi(1-\nu) r}\left[2 \delta_{q s} \hat{r}_{i} \hat{r}_{k}+2\left(\delta_{i k} \delta_{q s}-2 \nu \delta_{i s} \delta_{k q}-(1-\nu) \delta_{i q} \delta_{k s}\right)\right]
$$

The traction identity derived from (3), written in weak form, then reads

$$
\frac{1}{2}\langle\boldsymbol{p}, \tilde{\boldsymbol{u}}\rangle_{S}+\langle\mathcal{D}[S, \boldsymbol{u}], \tilde{\boldsymbol{u}}\rangle_{\partial \Omega}-\left\langle\mathcal{D}\left[S_{c}, \boldsymbol{\phi}\right], \tilde{\boldsymbol{u}}\right\rangle_{\partial \Omega}-\left\langle\mathcal{K}^{\prime}[S, \boldsymbol{p}], \tilde{\boldsymbol{u}}\right\rangle_{\partial \Omega}=0
$$

Assume boundary conditions in the form of traction-free cracks, prescribed tractions $\boldsymbol{p}^{\mathrm{D}}$ over the part $S_{p}$ of $S$ and prescribed displacements $\boldsymbol{u}^{\mathrm{D}}$ over the complementary part $S_{u}:=S \backslash S_{p}$. The SGBIE formulation is then obtained by combining (i) equation (5) with test functions $\tilde{\boldsymbol{p}}$ supported on $S_{u}$, (ii) equation (10) with test functions $\tilde{\boldsymbol{u}}$ supported on $S_{p}$, (iii) equation (10) with test functions $\tilde{\boldsymbol{u}}=\tilde{\boldsymbol{\phi}}$ supported on $S_{c}$, and introducing the boundary data $\boldsymbol{u}^{\mathrm{D}}, \boldsymbol{p}^{\mathrm{D}}$ into the resulting identities, to obtain the symmetric governing SGBIE formulation for the unknowns $\boldsymbol{u}, \boldsymbol{p}, \boldsymbol{\phi}$, whose respective supports are $S_{p}, S_{u}, S_{c}$ :

$$
\begin{aligned}
\left\langle\mathcal{V}\left[S_{u}, \boldsymbol{p}\right], \tilde{\boldsymbol{p}}\right\rangle_{S_{u}}-\left\langle\mathcal{K}\left[S_{p}, \boldsymbol{u}\right], \tilde{\boldsymbol{p}}\right\rangle_{S_{u}}+\left\langle\mathcal{W}\left[S_{c}, \boldsymbol{\phi}\right], \tilde{\boldsymbol{p}}\right\rangle_{S_{u}} & =\mathcal{F}_{p}(\tilde{\boldsymbol{p}}) \\
-\left\langle\mathcal{K}^{\prime}\left[S_{u}, \boldsymbol{p}\right], \tilde{\boldsymbol{u}}\right\rangle_{S_{p}}+\left\langle\mathcal{D}\left[S_{p}, \boldsymbol{u}\right], \tilde{\boldsymbol{u}}\right\rangle_{S_{p}}-\left\langle\mathcal{D}\left[S_{c}, \boldsymbol{\phi}\right], \tilde{\boldsymbol{u}}\right\rangle_{S_{p}} & =\mathcal{F}_{u}(\tilde{\boldsymbol{u}}) \\
\left\langle\mathcal{W}^{\prime}\left[S_{u}, \boldsymbol{p}\right], \tilde{\boldsymbol{\phi}}\right\rangle_{S_{c}}-\left\langle\mathcal{D}\left[S_{p}, \boldsymbol{u}\right], \tilde{\boldsymbol{\phi}}\right\rangle_{S_{c}}+\left\langle\mathcal{D}\left[S_{c}, \boldsymbol{\phi}\right], \tilde{\boldsymbol{\phi}}\right\rangle_{S_{c}} & =\mathcal{F}_{\phi}(\tilde{\boldsymbol{\phi}})
\end{aligned}
$$

with the linear forms $\mathcal{F}_{p}, \mathcal{F}_{u}, \mathcal{F}_{\phi}$ defined by

$$
\begin{aligned}
& \mathcal{F}_{p}(\tilde{\boldsymbol{p}})=\frac{1}{2}\left\langle\boldsymbol{u}^{\mathrm{D}}, \tilde{\boldsymbol{p}}\right\rangle_{S_{u}}+\left\langle\mathcal{K}\left[S_{u}, \boldsymbol{u}^{\mathrm{D}}\right], \tilde{\boldsymbol{p}}\right\rangle_{S_{u}}-\left\langle\mathcal{V}\left[S_{p}, \boldsymbol{p}^{\mathrm{D}}\right], \tilde{\boldsymbol{p}}\right\rangle_{S_{u}} \\
& \mathcal{F}_{u}(\tilde{\boldsymbol{u}})=-\frac{1}{2}\left\langle\boldsymbol{p}^{\mathrm{D}}, \tilde{\boldsymbol{u}}\right\rangle_{S_{p}}-\left\langle\mathcal{D}\left[S_{u}, \boldsymbol{u}^{\mathrm{D}}\right], \tilde{\boldsymbol{u}}\right\rangle_{S_{p}}+\left\langle\mathcal{K}^{\prime}\left[S_{p}, \boldsymbol{p}^{\mathrm{D}}\right], \tilde{\boldsymbol{u}}\right\rangle_{S_{p}} \\
& \mathcal{F}_{\phi}(\tilde{\boldsymbol{\phi}})=\left\langle\mathcal{D}\left[S_{u}, \boldsymbol{u}^{\mathrm{D}}\right], \tilde{\boldsymbol{\phi}}\right\rangle_{S_{c}}-\left\langle\mathcal{K}^{\prime}\left[S_{p}, \boldsymbol{p}^{\mathrm{D}}\right], \tilde{\boldsymbol{\phi}}\right\rangle_{S_{c}}
\end{aligned}
$$




\subsection{Solution strategy for the SGBEM equations}

The spaces $\mathcal{V}_{u}, \mathcal{V}_{p}, \mathcal{V}_{\phi}$ of admissible boundary traces are defined as

$$
\mathcal{V}_{u}=\left\{\boldsymbol{u} \in \tilde{H}^{1 / 2}\left(S ; \mathbb{R}^{3}\right)\right\}, \quad \mathcal{V}_{\phi}=\left\{\boldsymbol{\phi} \in \tilde{H}^{1 / 2}\left(S_{c} ; \mathbb{R}^{3}\right)\right\}, \quad \mathcal{V}_{p}=\left\{\boldsymbol{p} \in H^{-1 / 2}\left(S ; \mathbb{R}^{3}\right)\right\},
$$

and $\tilde{\boldsymbol{u}}, \tilde{\boldsymbol{\phi}}, \tilde{\boldsymbol{p}}$ are test displacements, displacement jumps, and tractions belonging to the same spaces (see e.g. [13] for the definition of these spaces). For Galerkin discretization, natural finitedimensional subspaces of $\mathcal{V}_{u}, \mathcal{V}_{\phi}$ consist of continuous interpolations of $\boldsymbol{u}$ over $S_{p}$ and $\boldsymbol{\phi}$ over $S_{c}$ with a zero trace on the edges $\partial S_{p}, \partial S_{c}$, while piecewise-continuous interpolation of $\boldsymbol{p}$ over $S_{u}$ define appropriate subspaces of $\mathcal{V}_{p}$. Note that the data $\boldsymbol{u}^{\mathrm{D}}$ appearing in the linear forms $\mathcal{F}_{u}$ and $\mathcal{F}_{t}$ is actually an arbitrary extension to $S$ of the displacement value prescribed on $S_{u}$ having $H^{1 / 2}(S)$ regularity (the corresponding integrals in $\mathcal{F}_{p}, \mathcal{F}_{u}, \mathcal{F}_{\phi}$ thus being taken over $S$ ), so that the actual displacement on $S_{p}$ is $\boldsymbol{u}+\boldsymbol{u}^{\mathrm{D}}$. This allows $\boldsymbol{u}$ and $\tilde{\boldsymbol{u}}$ to belong to the same space $\mathcal{V}_{u}$. The discretized set of equations arising from (11) is symmetric by virtue of the fact that the same interpolation spaces are used for the unknowns and trial functions, and has the form

$$
\mathbf{K X}=\mathbf{F}
$$

where the symmetric matrix $\mathbf{K} \in \mathbb{R}_{\mathrm{sym}}^{N \times N}$ arises from discretizing the bilinear forms, $\mathbf{X} \in \mathbb{R}^{N}$ collects all unknown DOFs on $S_{p}, S_{c}$ and $S_{u}$, and $\mathbf{F} \in \mathbb{R}^{N}$ corresponds to the right-hand side of (11). The operators $\mathcal{K}, \mathcal{K}^{\prime}$ are in practice reformulated in regularized form [36] to avoid actual evaluation of principal value integrals.

BEM problems of large size $N$ are solved by means of iterative algorithms such as GMRES [39]. Each GMRES iteration requires one evaluation of $\mathbf{K W}$ for given $\mathbf{W} \in \mathbb{R}^{N}$ (in practice, $\mathbf{W}$ and $\mathbf{K W}$ are two consecutive Krylov vectors). The fast multipole method (FMM) mainly aims at achieving a computational complexity lower than $O\left(N^{2}\right)$ for evaluating $\mathbf{K W}$, taking advantage of the low-rank approximation of submatrices of $\mathbf{K}$ implicitly achieved by the FMM. For the present elastostatic context, the expected computational complexity of one matrix-vector product evaluation is $O(N)$.

\subsection{Fast Multipole algorithm in BEM}

The FM-SGBEM is based on a reformulation of the tensorial kernels $\boldsymbol{U}, \boldsymbol{T}, \boldsymbol{B}$ into multipole expansions, which achieve a separation of the variables $\boldsymbol{x}$ and $\tilde{\boldsymbol{x}}$. For this purpose, the relative position vector $\boldsymbol{r}=\boldsymbol{x}-\tilde{\boldsymbol{x}}$ (Fig. 2) is decomposed into $\boldsymbol{r}=\boldsymbol{x}^{\prime}+\boldsymbol{r}_{0}-\tilde{\boldsymbol{x}}^{\prime}$ with $\boldsymbol{r}_{0}=\boldsymbol{x}_{0}-\tilde{\boldsymbol{x}}_{0}, \boldsymbol{x}^{\prime}=\boldsymbol{x}-\boldsymbol{x}_{0}$ and $\tilde{\boldsymbol{x}}^{\prime}=\tilde{\boldsymbol{x}}-\tilde{\boldsymbol{x}}_{0}$, where $\boldsymbol{x}_{0}, \tilde{\boldsymbol{x}}_{0}$ are two origins about which the expansions are performed. With these notations, the multipole expansion of $1 / r$ has the form (see e.g. [47])

$$
\frac{1}{r}=\lim _{p \rightarrow+\infty} \sum_{n=0}^{p} \sum_{m=-n}^{n}(-1)^{n} R_{n m}\left(\tilde{\boldsymbol{x}}^{\prime}\right) \sum_{n^{\prime}=0}^{n} \sum_{m^{\prime}=-n^{\prime}}^{n^{\prime}} \overline{S_{n+n^{\prime}, m+m^{\prime}}\left(\boldsymbol{r}_{0}\right)} R_{n^{\prime} m^{\prime}}\left(\boldsymbol{x}^{\prime}\right),
$$

where the (complex-valued) solid harmonics $R_{n m}(\boldsymbol{y}), S_{n m}(\boldsymbol{y})$ are defined and computable e.g. by means of known recursive relations [47]. For given poles $\boldsymbol{x}_{0}, \tilde{\boldsymbol{x}}_{0}$, expansion (13) is convergent for any $\boldsymbol{x}_{0}, \tilde{\boldsymbol{x}}_{0}$ such that:

$$
\left\|\boldsymbol{x}^{\prime}\right\|<\left\|\tilde{\boldsymbol{x}}^{\prime}-\boldsymbol{r}_{0}\right\| \text { and }\left\|\tilde{\boldsymbol{x}}^{\prime}\right\|<\left\|\boldsymbol{x}^{\prime}+\boldsymbol{r}_{0}\right\|
$$

The Kelvin solution $(1 \mathrm{a}, \mathrm{b})$ can then be rewritten in terms of $1 / r$ and its derivatives:

$$
\boldsymbol{U}(\boldsymbol{x}, \tilde{\boldsymbol{x}})=\frac{1}{16 \pi \mu(1-\nu)}\left[\boldsymbol{I}-(3-4 \nu)\left(\boldsymbol{x}+\boldsymbol{r}_{0}-\tilde{\boldsymbol{x}}\right) \otimes \boldsymbol{\nabla}\right] \frac{1}{r},
$$




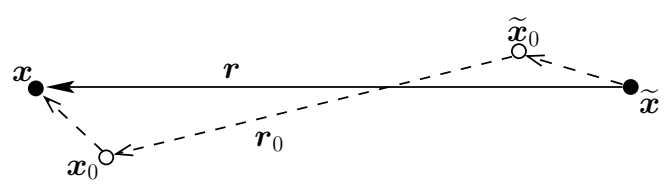

Figure 2: Decomposition of the position vector: notation

with similar reformulations also available for the other kernels $\boldsymbol{T}$ and $\boldsymbol{B}$. This allows to take advantage of (13) for deriving multipole expansions of the elastostatic kernels.

Now, consider two subsets $\Gamma\left(\boldsymbol{x}_{0}\right), \tilde{\Gamma}\left(\tilde{\boldsymbol{x}}_{0}\right)$ of $S$, such that (14) holds for any $\boldsymbol{x} \in \Gamma\left(\boldsymbol{x}_{0}\right)$ and $\tilde{\boldsymbol{x}} \in \tilde{\Gamma}\left(\tilde{\boldsymbol{x}}_{0}\right)$ with suitably chosen poles $\boldsymbol{x}_{0}, \tilde{\boldsymbol{x}}_{0}$ (such subsets are said to be well-separated). Then, the contribution of surfaces $\Gamma\left(\boldsymbol{x}_{0}\right), \tilde{\Gamma}\left(\tilde{\boldsymbol{x}}_{0}\right)$ to the bilinear form $\mathcal{B}_{p p}(\boldsymbol{p}, \tilde{\boldsymbol{p}})$, denoted by $B_{p p}\left(\boldsymbol{x}_{0}, \tilde{\boldsymbol{x}}_{0}\right)$ and given by

$$
B_{p p}\left(\boldsymbol{x}_{0}, \tilde{\boldsymbol{x}}_{0}\right):=\int_{\Gamma\left(\boldsymbol{x}_{0}\right)} \int_{\tilde{\Gamma}\left(\tilde{\boldsymbol{x}}_{0}\right)} \boldsymbol{p}(\boldsymbol{x}) \cdot \boldsymbol{U}(\boldsymbol{x}, \tilde{\boldsymbol{x}}) \cdot \tilde{\boldsymbol{p}}(\tilde{\boldsymbol{x}}) \mathrm{d} S_{\tilde{x}} \mathrm{~d} S_{x},
$$

can be evaluated by replacing the kernel $\boldsymbol{U}$ by its multipole expansion. Indeed, inserting expansion (13), truncated at a finite value of $p$, into (15) and exploiting the resulting expression of $\boldsymbol{U}$ in (16), $B_{p p}\left(\boldsymbol{x}_{0}, \tilde{\boldsymbol{x}}_{0}\right)$ is found to be given by

$$
B_{p p}\left(\boldsymbol{x}_{0}, \tilde{\boldsymbol{x}}_{0}\right)=\sum_{n=0}^{p} \sum_{m=-n}^{n}(-1)^{n}\left\{\widetilde{\boldsymbol{M}}_{n m}^{1}\left(\tilde{\boldsymbol{x}}_{0}\right) \cdot \boldsymbol{L}_{n m}^{1}\left(\boldsymbol{x}_{0}\right)+\widetilde{M}_{n m}^{2}\left(\tilde{\boldsymbol{x}}_{0}\right) L_{n m}^{2}\left(\boldsymbol{x}_{0}\right)\right\}+\epsilon_{p}
$$

(where $\epsilon_{p}$ is the truncation error resulting from using a finite value of $p$ in (13)), in terms of the multipole moments associated to the pole $\tilde{\boldsymbol{x}}_{0}$ :

$$
\begin{aligned}
\widetilde{\boldsymbol{M}}_{n m}^{1}\left(\tilde{\boldsymbol{x}}_{0}\right) & =\int_{\tilde{\Gamma}\left(\tilde{\boldsymbol{x}}_{0}\right)}[\boldsymbol{I}+(3-4 \nu) \tilde{\boldsymbol{x}} \otimes \boldsymbol{\nabla}] R_{n m}(\tilde{\boldsymbol{x}}) \cdot \tilde{\boldsymbol{p}}(\boldsymbol{x}) \mathrm{d} S_{\tilde{x}} \\
\widetilde{M}_{n m}^{2}\left(\tilde{\boldsymbol{x}}_{0}\right) & =(3-4 \nu) \int_{\tilde{\Gamma}\left(\tilde{\boldsymbol{x}}_{0}\right)} \boldsymbol{\nabla} R_{n m}(\tilde{\boldsymbol{x}}) \cdot \tilde{\boldsymbol{p}}(\tilde{\boldsymbol{x}}) \mathrm{d} S_{\tilde{x}}
\end{aligned}
$$

and the local expansion coefficients

$$
\begin{aligned}
& \boldsymbol{L}_{n m}^{1}\left(\boldsymbol{x}_{0}\right)=\sum_{n^{\prime}=0}^{n} \sum_{m^{\prime}=-n^{\prime}}^{n^{\prime}} \overline{S_{n+n^{\prime}, m+m^{\prime}}\left(\boldsymbol{r}_{0}\right)}\left[\boldsymbol{M}_{n^{\prime} m^{\prime}}^{1}\left(\boldsymbol{x}_{0}\right)+\boldsymbol{r}_{0} M_{n^{\prime} m^{\prime}}^{2}\left(\boldsymbol{x}_{0}\right)\right] \\
& L_{n m}^{2}\left(\boldsymbol{x}_{0}\right)=\sum_{n^{\prime}=0}^{n} \sum_{m^{\prime}=-n^{\prime}}^{n^{\prime}} \overline{S_{n+n^{\prime}, m+m^{\prime}}\left(\boldsymbol{r}_{0}\right)} M_{n^{\prime} m^{\prime}}^{2}\left(\boldsymbol{x}_{0}\right)
\end{aligned}
$$

defined in terms of the multipole moments associated to the pole $\boldsymbol{x}_{0}$ :

$$
\boldsymbol{M}_{n m}^{1}\left(\boldsymbol{x}_{0}\right)=\int_{\Gamma\left(\boldsymbol{x}_{0}\right)} R_{n m}(\boldsymbol{x}) \boldsymbol{p}(\boldsymbol{x}) \mathrm{d} S_{x}, \quad M_{n m}^{2}\left(\boldsymbol{x}_{0}\right)=\int_{\Gamma\left(\boldsymbol{x}_{0}\right)} R_{n m}(\boldsymbol{x})(\boldsymbol{x} \cdot \boldsymbol{p}(\boldsymbol{x})) \mathrm{d} S_{x}
$$

Similar treatments apply for the other bilinear forms involved in (11). The computation of contributions of type (16) for surface portions $\Gamma\left(\boldsymbol{x}_{0}\right), \tilde{\Gamma}\left(\tilde{\boldsymbol{x}}_{0}\right)$ which violate the separation conditions (14) need to be effected using the classical SGBEM quadrature methods; this normally includes all cases of (coincident, edge-adjacent or vertex-adjacent) singular integrations.

The usual way to define pairs of well-separated boundary portions $\Gamma\left(\boldsymbol{x}_{0}\right), \tilde{\Gamma}\left(\tilde{\boldsymbol{x}}_{0}\right)$ (so as to take advantage of (17) and similar expressions for the other SGBEM bilinear forms whenever permitted 
by the well-separatedness conditions (14)) consists in implementing a hierarchical subdivision of the region of space containing the boundary of interest $\partial \Omega$ into cubic cells. Each non-terminal cell (or non-leaf cell, in FMM parlance) is then subdivided into $2 \times 2 \times 2$ children cubic cells. Poles $\boldsymbol{x}_{0}$ or $\tilde{\boldsymbol{x}}_{0}$ are then taken as cell centers, and $\Gamma\left(\boldsymbol{x}_{0}\right)$ or $\tilde{\Gamma}\left(\tilde{\boldsymbol{x}}_{0}\right)$ as those portions of $\partial \Omega$ intersecting the cubic cell centered at $\boldsymbol{x}_{0}$ or $\tilde{\boldsymbol{x}}_{0}$. Following this approach, $\Gamma\left(\boldsymbol{x}_{0}\right)$ and $\tilde{\Gamma}\left(\tilde{\boldsymbol{x}}_{0}\right)$ satisfy the separation conditions (14) whenever the underlying same-level cells are not adjacent. The hierarchical subdivision aims at producing new pairs of non-adjacent children cells (at a lower level) whenever two parent cells are adjacent, thus reducing the cases where double integrals need to be computed by "traditional" (and costlier) methods. These considerations are at the root of the well-known multi-level fast multipole method, used here and described in more detail (for the present elastostatic SGBEM context) in [36]. It implicitly recasts the SGBEM system (12) in the form

$$
\mathbf{K}^{\mathrm{FMM}} \mathbf{X}+\mathbf{K}^{\text {near }} \mathbf{X}=\mathbf{F}
$$

where $\mathbf{K}^{\mathrm{FMM}}$ and $\mathbf{K}^{\text {near }}$ gather the contributions to $\mathbf{K}$ arising from pairs of cells that are wellseparated or adjacent, respectively. Importantly, the matrix $\mathbf{K}^{\mathrm{FMM}}$ is neither computed nor stored, as the iterative solver only exploits matrix-vector products (whose evaluation is in fact the main computational task effected by the FMM). In contrast, setting up and storing $\mathbf{K}^{\text {near }}$ (as a sparse matrix) is feasible (although not mandatory), see Sec. 3.1.

\section{Performance improvement}

Before addressing multizone configurations in Sec. 4, we consider two improvements of the FMSGBEM aiming at enhancing its computational efficiency. The first one concerns the evaluation and storage of the near-field influence matrix $\mathbf{K}^{\text {near }}$, taking advantage of its symmetry and sparsity (Sec. 3.1), while the second one consists in iteratively solving system (12) by means of the flexible GMRES preconditioned by $\mathbf{K}^{\text {near }}$, aiming to reduce the iteration count entailed by the linear solver (Sec. 3.2). Numerical experiments on a cracked single-region configuration using this modified version of the FM-SGBEM are then reported in Sec. 3.3.

\subsection{Evaluation and storage of near-field influence terms}

Evaluation. The evaluation of all contributions to the near component $\mathbf{K}^{\text {near }}$ of the SGBEM governing matrix must be done using traditional numerical quadrature methods, entailing significant computational work. To achieve a trade-off between accuracy and speed, the nonsingular double element integrals assembled into the $\mathbf{K}^{\text {near }}(C)$ are computed using Gaussian quadrature rules whose density is adjusted according to the spatial arrangement of the two elements involved. An empirical scheme for this purpose has been proposed in [37], whereby an indicator of severity (IS) that depends primarily on the ratio of the element size to the inter-element distance is computed:

$$
\text { IS }:=\lfloor(2.87+0.424 \cos \theta) H / d\rfloor,
$$

where $\lfloor\cdot\rfloor$ denotes the integral part of a number, the element size $H$ is defined (for the quadrilateral elements employed in this work) as the length of the longest diagonal, while $d$ refers to the distance between the element centers and $\theta$ is the angle between the line joining the element centers and the normal of the exterior element (Fig.3). The Gaussian rule is then selected according to the value of IS as shown in Table 1. Even though the cheapest $2 \times 2$ Gaussian rule ends up being used for over $80 \%$ of the element quadratures, the solution accuracy is found to be only marginally affected (compared to using $4 \times 4$ Gaussian rules throughout). 


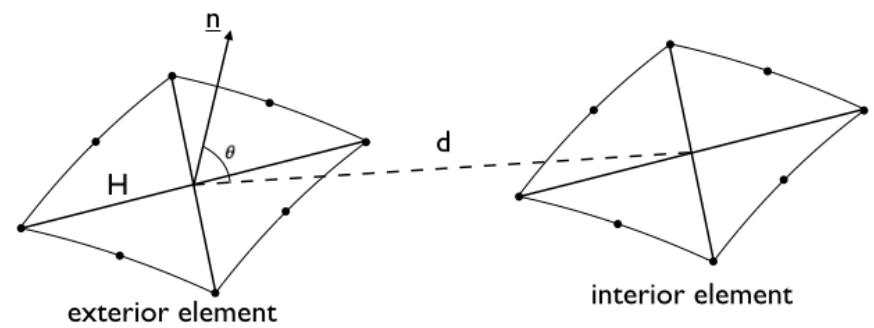

Figure 3: Geometry of a pair of elements

\begin{tabular}{ccccccccc}
\hline IS & 1 & 2 & 3 & 4 & 5 & 6 & 7 & 8 \\
Number of Gauss points & $2 \times 2$ & $3 \times 3$ & $4 \times 4$ & $5 \times 5$ & $6 \times 6$ & $4 \times(4 \times 4)$ & $4 \times(5 \times 5)$ & $4 \times(6 \times 6)$ \\
\hline
\end{tabular}

Table 1: Correspondence between the Indicator of Severity (IS) and the quadrature rule over one element [37]

Storage. The near influence contributions arise from pairs of adjacent cells, one of which at least being a leaf cell. For a given cell $C$, let $\mathcal{A}(C)$ denote the set of same-level cells that are adjacent to $C$ (with the convention that $C \in \mathcal{A}(C)$ ), noting that $\mathcal{A}(C)$ may contain up to 27 cells, and consider as an example the near-interaction contributions arising from $\mathcal{B}_{p p}$. Those result from the BEM discretization of either

$$
\int_{S \cap C} \sum_{C^{\prime} \in \mathcal{A}(C)} \int_{C^{\prime} \cap S} \boldsymbol{p}(\boldsymbol{x}) \cdot \boldsymbol{U}(\boldsymbol{x}, \tilde{\boldsymbol{x}}) \cdot \tilde{\boldsymbol{p}}(\tilde{\boldsymbol{x}}) \mathrm{d} S_{\tilde{x}} \mathrm{~d} S_{x}
$$

(if $C$ is a leaf cell) or

$$
\int_{S \cap C} \sum_{C^{\prime} \in \mathcal{A}(C), C^{\prime} \text { leaf }} \int_{C^{\prime} \cap S} \boldsymbol{p}(\boldsymbol{x}) \cdot \boldsymbol{U}(\boldsymbol{x}, \tilde{\boldsymbol{x}}) \cdot \tilde{\boldsymbol{p}}(\tilde{\boldsymbol{x}}) \mathrm{d} S_{\tilde{x}} \mathrm{~d} S_{x}
$$

(if $C$ is not leaf cell). Let $\mathbf{K}^{\text {near }}(C)$ gather the contribution to $\mathbf{K}^{\text {near }}$ produced by the BEM discretization of $(24 a, b)$ and the corresponding contributions of the other SGBIE bilinear forms (note that $(24 \mathrm{~b})$ yields a zero contribution whenever $\mathcal{A}(C)$ contains no leaf cell). With these conventions (and treating all matrices $\mathbf{K}^{\text {near }}(C)$ as extended to the whole SGBEM model), one has

$$
\mathbf{K}^{\text {near }}=\sum_{C} \mathbf{K}^{\text {near }}(C)
$$

with the sum going over all nonempty cells $C$. Each submatrix $\mathbf{K}^{\text {near }}(C)$ is symmetric and sparse, the sparsity resulting from the fact that only contributions from $C$ and some of its adjacent cells are involved in (24a,b), as sketched in Fig. 4.

To take advantage of the symmetry and sparsity of each $\mathbf{K}^{\text {near }}(C)$, a simple storage algorithm called symmetric compressed sparse row can be used, whereby the upper-triangular part of a symmetric sparse matrix is stored as three linear arrays $A A, J A, I A$ containing respectively the non-zero entries, the column indexes and the position in AA of the first non-zero entry of each row. Summarizing, the adopted storage scheme for $\mathbf{K}^{\text {near }}$ facilitates tackling large FM-SGBEM models with moderate resources: (i) the global $\mathbf{K}^{\text {near }}$ is decomposed into cell-wise blocks $\mathbf{K}^{\text {near }}(C)$ of moderate sizes; (ii) each block is efficiently compressed, thus further reducing the required memory. 

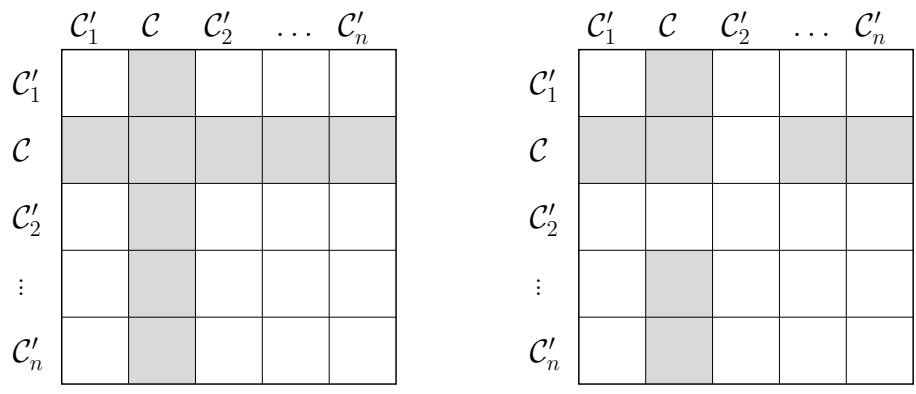

Figure 4: Block-wise population of $\mathbf{K}^{\text {near }}(C)$ for cases (24a) ( $C$ is a leaf, left) and (24b) ( $C$ is not a leaf, right), with grey squares representing nonzero blocks. There are $n+1$ cells in $\mathcal{A}(C)$ (with $n \leq 26$ for $3 \mathrm{D}$ problems). In case (24a), interactions of $C$ with all $C^{\prime} \in \mathcal{A}(C)$ are computed, while in case (24b) only the leaf cells $C^{\prime}$ in $\mathcal{A}(C)$ are involved.

\subsection{Flexible GMRES}

The second concern is to reduce the iterative solution time, which is done mainly by using a preconditioned version of the iterative solver GMRES, in order to lower the iteration count. Many preconditioning strategies have been proposed, exploiting e.g. incomplete LU (ILU) factorization [41], sparse approximate inverses (SPAI) [32, 11], Calderon identities [43] or on-surface radiation condition (OSRC) [14]. In addition, the strategy where a low-precision inner GMRES solver to the near-interaction matrix $\mathbf{K}^{\text {near }}$ is used as a right preconditioner is simple to implement and has proved to be effective when applied to the elastodynamic collocation FM-BEM [8]. This scheme, which is a particular instance of a flexible GMRES (F-GMRES) solver [38], is adopted in this work. The major iteration loop produces, as usual with GMRES solvers, a sequence of nested subspaces of $\mathbb{R}^{N}$ that are generated by Krylov vectors $\mathbf{W}_{k}$ such that

$$
\mathbf{K}^{\text {near }} \mathbf{W}_{k+1}=\mathbf{K W}_{k}
$$

The main computational task involved in the iterative solution algorithm lies in evaluating the matrix-vector product $\mathbf{Y}_{k}:=\mathbf{K W}_{k}$ (one such evaluation per main GMRES iteration). The preconditioning then consists in solving the above system of equations each time a new Krylov vector is computed, using in this work a low-precision GMRES solver (the stopping criterion being of the form $\left\|\mathbf{K}^{\text {near }} \mathbf{W}_{k+1}-\mathbf{Y}_{k}\right\| \leq \epsilon_{2}\left\|\mathbf{Y}_{k}\right\|$, with typically $\left.\epsilon_{2}=10^{-1}\right)$. This inner GMRES solver is itself enhanced by a simple diagonal preconditioning. The outer GMRES iterations are carried out until the condition $\|\mathbf{F}-\mathbf{B X}\| \leq \epsilon_{1}\|\mathbf{F}\|$ is met (with typically $\epsilon_{1}=10^{-3}$ ).

\subsection{Numerical validation}

The numerical code is written in Fortran 90. All numerical experiments reported hereafter are run on a single-processor PC (3Ghz, 48Gb RAM). In this example, the FM-SGBEM is applied to an array of $n_{c} \times n_{c} \times n_{c}$ randomly-oriented penny-shaped cracks of radius $r_{c}=1$ (Fig. 5), whose centers are located at the nodes of a cubic grid with regular spacing $d_{c}=4$ oriented along the coordinate axes. The array is embedded in an unbounded homogeneous domain, characterized by $E=1, \nu=0.3846$ and subjected to a remote tensile load $\sigma_{33}^{0}=1$. Each crack is modelled using 48 eight-noded quadrilateral elements. The elements adjacent to the crack front are quarter-point elements [22]. We compute the COD by solving the following variational traction equation:

$$
\mathcal{B}_{\phi \phi}(\boldsymbol{\phi}, \tilde{\boldsymbol{\phi}})=\left\langle\boldsymbol{\sigma}^{0} \cdot \boldsymbol{n}, \tilde{\boldsymbol{\phi}}\right\rangle_{S_{c}} \quad \forall \tilde{\boldsymbol{\phi}} \in \mathcal{V}_{c}
$$




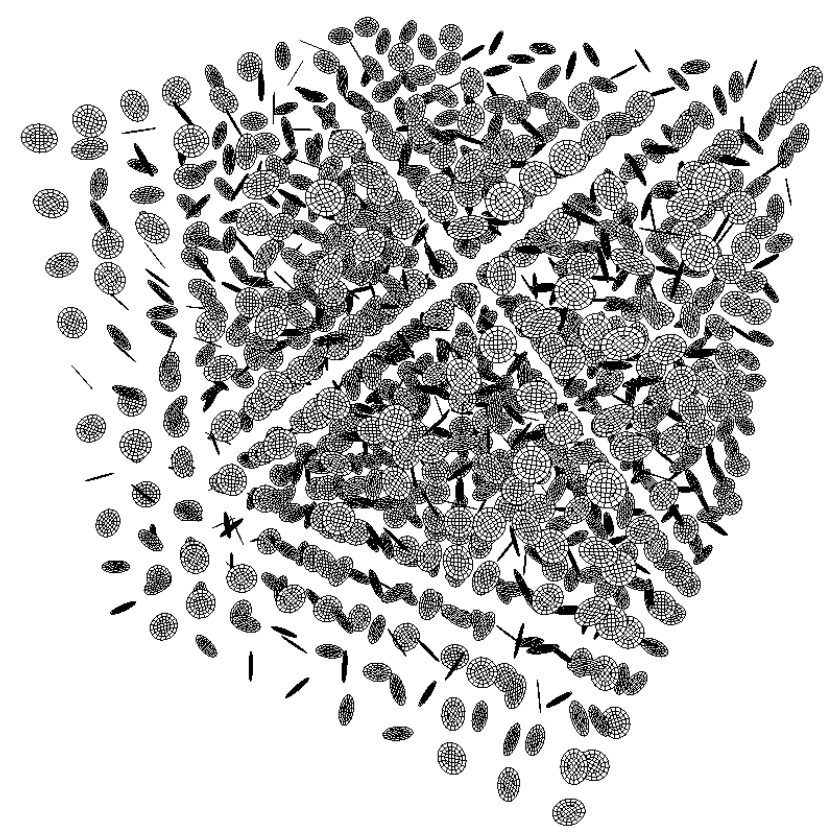

Figure 5: Array of randomly oriented penny-shaped cracks embedded in an unbounded domain $\left(n_{c}=10\right)$.

\begin{tabular}{|c||c|c|c|cc|cc|cc|cc|}
\hline \multirow{2}{*}{ Mesh } & $N$ & $\ell$ & $M$ & \multicolumn{2}{|c|}{ iters } & \multicolumn{2}{c|}{ CPU (s) / iter. } & \multicolumn{2}{c|}{ CPU (s), GMRES } & \multicolumn{2}{c|}{ CPU (s), total } \\
\cline { 5 - 11 } & & & & GMRES & Flex & GMRES & Flex & GMRES & F-GMRES & GMRES & F-GMRES \\
\hline M1 & $1,061,928$ & 7 & 15 & 18 & 6 & 1,569 & 1,626 & 28,217 & 11,155 & 38,952 & 21,807 \\
M2 & $1,585,152$ & 6 & 30 & 18 & 4 & 2,317 & 2,349 & 42,659 & 11,706 & 57,067 & 26,053 \\
M3 & $2,256,984$ & 7 & 30 & 18 & 4 & 3,299 & 3,377 & 59,004 & 16,711 & 84,904 & 42,424 \\
M4 & $3,096,000$ & 7 & 30 & 18 & 4 & 4,576 & 4,756 & 79,403 & 23,542 & 128,828 & 73,383 \\
\hline
\end{tabular}

Table 2: Crack array under remote tension: computational data for FM-SGBEM solutions using GMRES or FGMRES ( $\bar{\ell}$ and $M$ respectively denote the octree depth and the maximum number of elements in a leaf cell).

The performance of F-GMRES is tested on four meshes M1, M2, M3, M4, respectively corresponding to $n_{c}=14,16,18,20$, by applying the FM-SGBEM formulation of (25) in conjunction with either (i) GMRES left-preconditioned by the diagonal of $\mathbf{K}^{\text {near }}$ or (ii) F-GMRES rightpreconditioned by $\mathbf{K}^{\text {near }}$. Since the computation of $\mathbf{K}^{\text {near }}$ and $\mathbf{F}$ proceeds identically for both solvers, their respective runtimes are only affected by the iterative solution part of the computation. Computational data for these tests, given in Table 2, shows that F-GMRES achieves a significant reduction of the solution times (of up to over 70\%) relative to standard GMRES, through much-reduced lower iteration counts. Moreover, the CPU time per iteration is seen in both cases to depend linearly on the problem size $N$, consistently with the well-known linear complexity predicted by theoretical studies of the FMM. For this example, the GMRES iteration count is observed to be virtually independent on $N$. This is believed to result from the fact that the boundary is made of many separated objects (the cracks), making $\mathbf{K}^{\text {near }}$ the block diagonal matrix that treats each crack as isolated in an uncracked infinite medium. Such a matrix is a very good preconditioner. The very low and model size-independent iteration counts reported in [30] for the FMM treatment of many-inclusions problems corroborate this interpretation. 


\section{FM-SGBEM in multizone problems}

\subsection{Multizone formulation}

Considering a fractured solid $\Omega$ made of $L$ homogeneous sub-domains $\Omega^{\ell}(1 \leq \ell \leq L)$, see Fig. 6 . Each subdomain may contain internal cracks, defined by the open surface $S_{c}^{\ell}$. The boundary $S^{\ell}$ of $\Omega^{\ell}$ may be partitioned according to

$$
\partial \Omega^{\ell}=S_{c}^{\ell} \cup S_{u}^{\ell} \cup S_{p}^{\ell} \cup \Sigma^{\ell},
$$

where $S_{u}^{\ell}:=S_{u} \cap \partial \Omega^{\ell}$ and $S_{p}^{\ell}:=S_{p} \cap \partial \Omega^{\ell}$ are the contributions of $\partial \Omega^{\ell}$ to the Dirichlet and Neumann surfaces, and $\Sigma^{\ell}=\partial \Omega^{\ell} \backslash \partial \Omega$ is the interfacial part of $\partial \Omega^{\ell}$, i.e. the part shared with the boundaries of neighboring subregions. Moreover, let $\Sigma^{\ell, m}:=\partial \Omega^{\ell} \cap \partial \Omega^{m}$ denote the interface shared by two adjacent subdomains $\Omega^{\ell}, \Omega^{m}$ (implying, in particular, that $\Sigma^{\ell, m} \subset \Sigma^{\ell}$ and $\Sigma^{\ell, m} \subset \Sigma^{m}$ ). On any such interface, both the displacement and the traction are unknown.

The governing SGBIE formulation for a given subdomain $\Omega^{\ell}$ is obtained from (11) by replacing $S_{u}, S_{p}, S_{c}$ with $S_{u}^{\ell} \cup \Sigma^{\ell}, S_{p}^{\ell} \cup \Sigma^{\ell}, S_{c}^{\ell}$, and reads

$$
\begin{array}{r}
\left\langle\mathcal{V}\left[S_{u}^{\ell} \cup \Sigma^{\ell}, \boldsymbol{p}^{\ell}\right], \tilde{\boldsymbol{p}}\right\rangle_{S_{u}^{\ell} \cup \Sigma^{\ell}}-\frac{1}{2}\left\langle\boldsymbol{u}^{\ell}, \tilde{\boldsymbol{p}}\right\rangle_{\Sigma^{\ell}}-\left\langle\mathcal{K}\left[S_{p}^{\ell} \cup \Sigma^{\ell}, \boldsymbol{u}^{\ell}\right], \tilde{\boldsymbol{p}}\right\rangle_{S_{u}^{\ell} \cup \Sigma^{\ell}}+\left\langle\mathcal{K}\left[S_{c}^{\ell}, \boldsymbol{\phi}^{\ell}\right], \tilde{\boldsymbol{p}}\right\rangle_{S_{u}^{\ell} \cup \Sigma^{\ell}}=\mathcal{F}_{p}^{\ell}(\tilde{\boldsymbol{p}}), \\
\frac{1}{2}\left\langle\boldsymbol{p}^{\ell}, \tilde{\boldsymbol{u}}\right\rangle_{\Sigma^{\ell}}-\left\langle\mathcal{K}^{\prime}\left[S_{u}^{\ell} \cup \Sigma^{\ell}, \boldsymbol{p}^{\ell}\right], \tilde{\boldsymbol{u}}\right\rangle_{S_{p}^{\ell} \cup \Sigma^{\ell}}+\left\langle\mathcal{D}\left[S_{p}^{\ell} \cup \Sigma^{\ell}, \boldsymbol{u}^{\ell}\right], \tilde{\boldsymbol{u}}\right\rangle_{S_{p}^{\ell} \cup \Sigma^{\ell}}-\left\langle\mathcal{D}\left[S_{c}^{\ell}, \boldsymbol{\phi}^{\ell}\right], \tilde{\boldsymbol{u}}\right\rangle_{S_{p}^{\ell} \cup \Sigma^{\ell}}=\mathcal{F}_{u}^{\ell}(\tilde{\boldsymbol{u}}) \\
\left\langle\mathcal{K}^{\prime}\left[S_{u}^{\ell} \cup \Sigma^{\ell}, \boldsymbol{p}^{\ell}\right], \tilde{\boldsymbol{\phi}}\right\rangle_{S_{c}^{\ell}}-\left\langle\mathcal{D}\left[S_{p}^{\ell} \cup \Sigma^{\ell}, \boldsymbol{u}^{\ell}\right], \tilde{\boldsymbol{\phi}}\right\rangle_{S_{c}^{\ell}}+\left\langle\mathcal{D}\left[S_{c}^{\ell}, \boldsymbol{\phi}^{\ell}\right], \tilde{\boldsymbol{\phi}}\right\rangle_{S_{c}^{\ell}}=\mathcal{F}_{\phi}^{\ell}(\tilde{\boldsymbol{\phi}}),
\end{array}
$$

where $\boldsymbol{u}^{\ell}, \boldsymbol{p}^{\ell}, \boldsymbol{\phi}^{\ell}$ are the boundary unknowns relative to $\Omega^{\ell}$ and with the linear forms $\mathcal{F}_{p}^{\ell}, \mathcal{F}_{u}^{\ell}, \mathcal{F}_{\phi}^{\ell}$ defined by

$$
\begin{aligned}
& \mathcal{F}_{p}^{\ell}(\tilde{\boldsymbol{p}})=\frac{1}{2}\left\langle\boldsymbol{u}^{\mathrm{D}}, \tilde{\boldsymbol{p}}\right\rangle_{S_{u}^{\ell}}+\left\langle\mathcal{K}\left[S^{\ell}, \boldsymbol{u}^{\mathrm{D}}\right], \tilde{\boldsymbol{p}}\right\rangle_{S_{u}^{\ell} \cup \Sigma^{\ell}}-\left\langle\mathcal{V}\left[S_{p}^{\ell}, \boldsymbol{p}^{\mathrm{D}}\right], \tilde{\boldsymbol{p}}\right\rangle_{S_{u}^{\ell} \cup \Sigma^{\ell}} \\
& \mathcal{F}_{u}^{\ell}(\tilde{\boldsymbol{u}})=-\frac{1}{2}\left\langle\boldsymbol{p}^{\mathrm{D}}, \tilde{\boldsymbol{u}}\right\rangle_{S_{p}^{\ell}}-\left\langle\mathcal{D}\left[S^{\ell}, \boldsymbol{u}^{\mathrm{D}}\right], \tilde{\boldsymbol{u}}\right\rangle_{S_{p}^{\ell} \cup \Sigma^{\ell}}+\left\langle\mathcal{K}^{\prime}\left[S_{p}^{\ell}, \boldsymbol{p}^{\mathrm{D}}\right], \tilde{\boldsymbol{u}}\right\rangle_{S_{p}^{\ell} \cup \Sigma^{\ell}} \\
& \mathcal{F}_{\phi}^{\ell}(\tilde{\boldsymbol{\phi}})=\left\langle\mathcal{D}\left[S, \boldsymbol{u}^{\mathrm{D}}\right], \tilde{\boldsymbol{\phi}}\right\rangle_{S_{c}}-\left\langle\mathcal{K}^{\prime}\left[S_{p}^{\ell}, \boldsymbol{p}^{\mathrm{D}}\right], \tilde{\boldsymbol{\phi}}\right\rangle_{S_{c}}
\end{aligned}
$$

A global SGBIE formulation for the complete cracked multiregion configuration is then obtained by invoking the SGBIE systems (26) for all subdomains and enforcing the perfect-bonding transmission conditions $\boldsymbol{u}^{\ell}=\boldsymbol{u}^{m}$ (displacement continuity) and $\boldsymbol{p}^{\ell}=-\boldsymbol{p}^{m}$ (equilibrium) linking the boundary traces on all interfaces $\Sigma^{\ell, m}$. The latter task can be effected by either (i) appending explicitly the set of all transmission conditions to the set of all SGBIE systems (26) or (ii) using the transmission conditions for eliminating redundant interfacial unknowns.

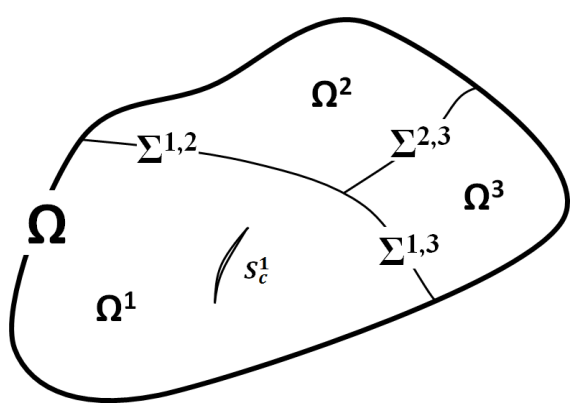

Figure 6: A multizone fractured domain 
Approach (ii) is followed here (and also in e.g. [21, 18]). For any pair of generic subregion numbers $1 \leq \ell<m \leq L$, let the interface $\Sigma^{\ell, m}=\Sigma^{m, \ell}$ be given a global interface number $I(\ell, m)$ if nonempty, i.e. if $\Omega^{\ell}$ and $\Omega^{m}$ are adjacent (that interface may then be denoted $\Sigma^{I(\ell, m)}$ ). Moreover, the normal vector on $\Sigma^{I(\ell, m)}$ is conventionally directed from $\Omega^{\ell}$ to $\Omega^{m}$. Then, a displacement field is defined over the whole system of surfaces and interfaces, together with corresponding test functions $\tilde{\boldsymbol{u}}$, with the provision that $\boldsymbol{u}, \tilde{\boldsymbol{u}}$ be continuous between connected surfaces and interfaces (a requirement achieved in practice by means of $C^{0}$-conforming boundary elements). Moreover, on each nonempty interface, one unknown traction field $\boldsymbol{p}^{I(\ell, m)}$ is defined, together with corresponding test functions $\tilde{\boldsymbol{u}}^{I(\ell, m)}, \tilde{\boldsymbol{p}}^{I(\ell, m)}$. The unknown and test displacements and tractions on $\Sigma^{I(\ell, m)}$ relative to $\Omega^{\ell}$ to $\Omega^{m}$ are then taken as

$$
\begin{array}{ll}
\boldsymbol{u}^{\ell}=\boldsymbol{u}^{m}=\left.\boldsymbol{u}\right|_{\Sigma^{I(\ell, m)}}, & \boldsymbol{p}^{\ell}=-\boldsymbol{p}^{m}=\boldsymbol{p}^{I(\ell, m)}, \\
\tilde{\boldsymbol{u}}^{\ell}=\tilde{\boldsymbol{u}}^{m}=\left.\tilde{\boldsymbol{u}}\right|_{\Sigma^{I(\ell, m)},} & \tilde{\boldsymbol{p}}^{\ell}=-\tilde{\boldsymbol{p}}^{m}=\tilde{\boldsymbol{p}}^{I(\ell, m)} .
\end{array}
$$

The SGBIE formulation for the complete multizone problem is then obtained by (i) expressing all unknowns and test functions on the interfaces using $(27 \mathrm{a}, \mathrm{b})$ in terms of the corresponding interfacebound quantities, and (ii) summing equations (26) written for all subregions. Equations (26) for a given subdomain are symmetric except for the single-integral terms $\left\langle\boldsymbol{u}^{\ell}, \tilde{\boldsymbol{p}}\right\rangle_{\Sigma^{\ell}}$ and $\left\langle\boldsymbol{p}^{\ell}, \tilde{\boldsymbol{u}}\right\rangle_{\Sigma^{\ell}}$. However, by virtue of $(27 \mathrm{a}, \mathrm{b})$, the latter can be checked to cancel out with similar contributions coming from adjacent subdomains, ensuring symmetry of the global multizone SGBIE formulation.

The SGBEM discretization divides all external, crack and interfacial surfaces into boundary elements. A $C^{0}$ conforming interpolation of all displacement-like quantities $\boldsymbol{u}, \boldsymbol{\phi}, \tilde{\boldsymbol{u}}, \tilde{\boldsymbol{\phi}}$ is assumed, so that in particular their continuity is enforced between all connected boundary and interface components. On the other hand, the interpolation of the traction-like quantities $\boldsymbol{p}, \tilde{\boldsymbol{p}}$ must allow for discontinuities between connected surface and interface components, in order to permit implementation of the sign conventions adopted in $(27 \mathrm{a}, \mathrm{b})$. We note [12] that the set of interfaces in a multi-region configuration is in general not orientable, i.e. it is not possible to define an orientation convention for the normal to the interfaces such that the normal to any given subdomain $\Omega^{\ell}$ has a uniform (either inward or outward) orientation over all of $\partial \Omega^{\ell}$, hence the necessity of adopting conventions such as $(27 \mathrm{a}, \mathrm{b})$.

The discretized counterpart of equations (26) yields a SGBEM linear system of the form

$$
\mathbf{K}^{\ell} \mathbf{X}^{\ell}=\mathbf{F}^{\ell}
$$

where (i) $\mathbf{X}^{\ell}$ gathers all boundary and interfacial unknowns pertaining to subdomain $\Omega^{\ell}$, (ii) $\mathbf{F}^{\ell}$ results from the discretization of the linear forms $\mathcal{F}_{p}^{\ell}, \mathcal{F}_{u}^{\ell}, \mathcal{F}_{\phi}^{\ell}$, and (iii) the matrix $\mathbf{K}^{\ell}$ is symmetric after disregarding the contributions from $\left\langle\boldsymbol{u}^{\ell}, \tilde{\boldsymbol{p}}\right\rangle_{\Sigma^{\ell}}$ and $\left\langle\boldsymbol{p}^{\ell}, \tilde{\boldsymbol{u}}\right\rangle_{\Sigma^{\ell}}$, known to cancel out in the global SGBEM system. The latter is then obtained by simply summing all equations (28), wherein matrices and vectors are understood as being extended to the whole multizone SGBEM model by means of appropriate zero-padding:

$$
\mathbf{K X}=\mathbf{F}, \quad \text { with } \mathbf{K}:=\sum_{\ell=1}^{L} \mathbf{K}^{\ell}, \quad \mathbf{F}:=\sum_{\ell=1}^{L} \mathbf{F}^{\ell}
$$

\subsection{Fast multipole treatment of the multizone problem}

Equation (29) indicates that the single-domain FM-SGBEM can be used as a building block for solving multizone problems, by the simple expedient of looping over the subdomains, evaluating for each $\Omega^{\ell}$ the contribution $\mathbf{F}^{\ell}$ to $\mathbf{F}$ (at the initialization stage of GMRES) and the contribution 


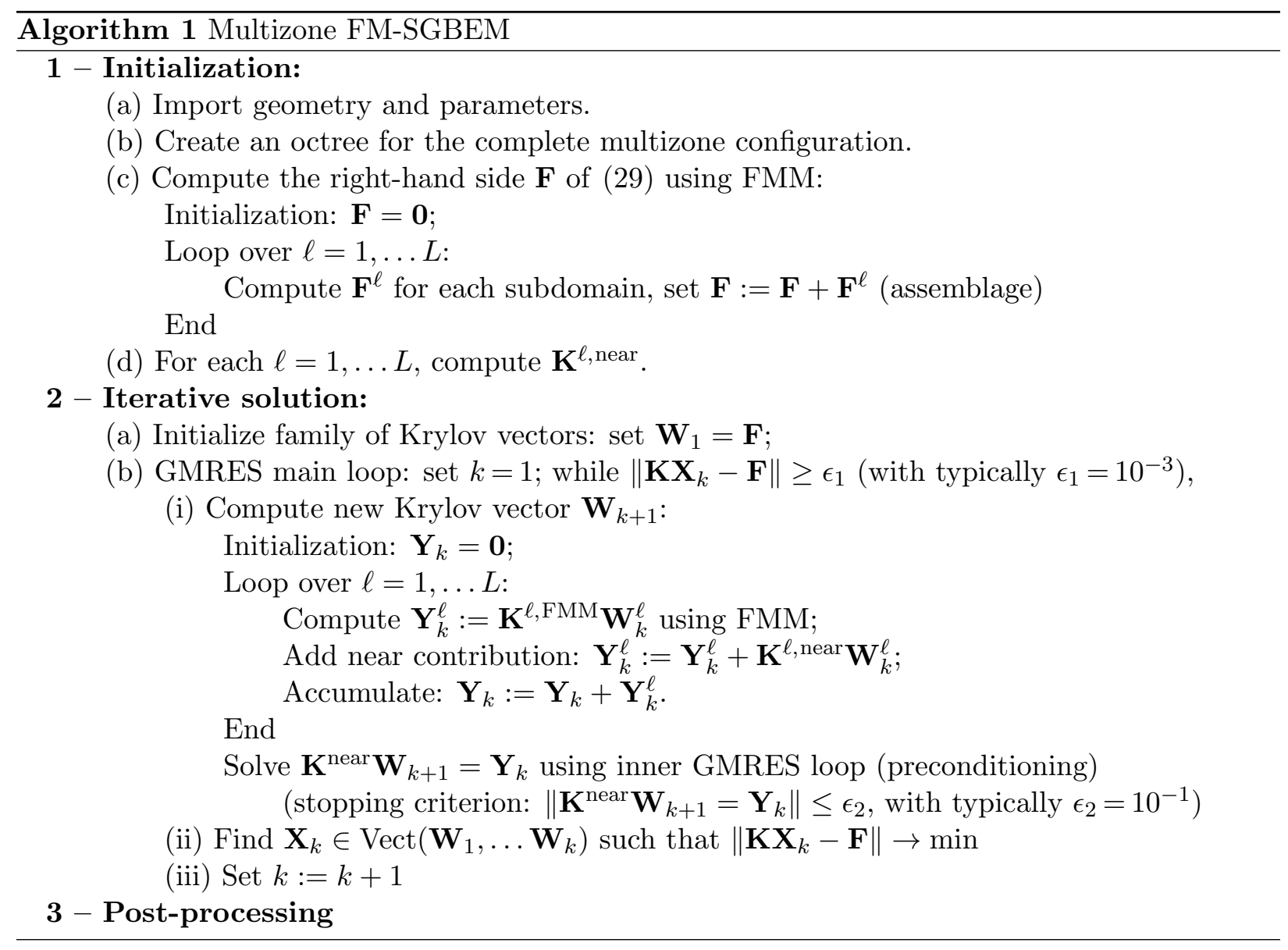

$\mathbf{Y}_{k}^{\ell}:=\mathbf{K}^{\ell} \mathbf{W}_{k}^{\ell}$ to $\mathbf{Y}_{k}:=\mathbf{K W}_{k}$ (when GMRES requires the $(k+1)$-th Krylov vector $\mathbf{W}_{k+1}$ ), with the global quantities $\mathbf{F}, \mathbf{Y}_{k}$ computed using an assembly-like procedure. Based on this observation, the main steps of the multizone FM-SGBEM are as shown in Algorithm 1. The Krylov vectors $\widetilde{\mathbf{W}}_{k}$ used in the inner GMRES loop (preconditioning step) are computed as

$$
\widetilde{\mathbf{W}}_{1}=\mathbf{Y}_{k}, \quad \widetilde{\mathbf{W}}_{k+1}=\sum_{\ell=1}^{L} \mathbf{K}^{\ell, \text { near }} \widetilde{\mathbf{W}}_{k}^{\ell},
$$

with the summation again performed by means of a loop over the subregions and accumulation.

The memory usage of multizone FM-SGBEM is similar to that of the single-domain counterpart. Near interactions are stored in sparse matrices $\mathbf{K}^{\ell, \text { near }}$; multipole moments are allocated in temporary spaces (corresponding to octree cells) that are freed when switching to another subdomain for performing the next FMM computation in the course of a loop over subdomains.

\section{Numerical examples}

The proposed multizone and multicrack SGBEM formulation is now tested on four examples. The first two examples (Secs. 5.1 and 5.2) are simple correctness tests on moderate-scale models. The last two examples (Secs. 5.3 and 5.4) demonstrate the formulation on large-scale BEM models. 


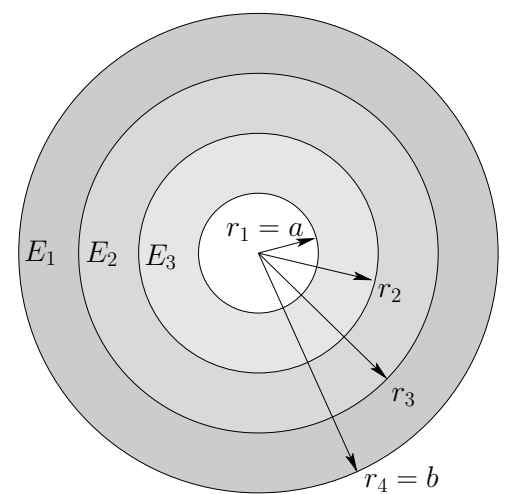

Figure 7: Three-layer spherical envelope under internal uniform pressure: $r_{1}=1, r_{2}=2, r_{3}=3, r_{4}=4$, materials: $E_{1}=1, E_{2}=2, E_{3}=3, \nu_{1}=\nu_{2}=\nu_{3}=0.3$.

\begin{tabular}{|l|cccc|}
\hline position & $r_{1}$ & $r_{2}$ & $r_{3}$ & $r_{4}$ \\
\hline relative error (\%) on $u_{r}$ & 0.50 & 0.90 & 0.92 & 0.92 \\
relative error (\%) on $t_{r}=\sigma_{r r}$ & - & -0.19 & -0.49 & - \\
\hline
\end{tabular}

Table 3: Spherical envelope under internal pressure: relative solution errors on surfaces and interfaces

\subsection{Spherical envelope under internal pressure}

The first numerical test consists of a spherical envelope made of three concentric material layers, of internal radius $a$ and external radius $b$. The constitutive material in each layer is elastic isotropic (with material parameters given in Fig. 7). The internal surface $r=r_{1}=a$ is subjected to a normal uniform pressure $p=1$, the outer surface $r=r_{4}=b$ is traction-free, and perfect bonding is assumed at the material interfaces $r=r_{2}$ and $r=r_{3}$. This simple test has an analytical solution, which for the radial displacement $u_{r}$ in the $i$-th layer is of the form $u_{r}(r)=A_{i} r+B_{i} r^{-2}$, the constants $A_{i}, B_{i}$ being determined by satisfying the boundary conditions $\sigma_{r r}(a)=-p, \sigma_{r r}(b)=0$ and the perfect bonding conditions at the interfaces $r=r_{2}$ and $r=r_{3}$.

In this example, the two external surfaces and the two interfaces are modelled using a total of 1047 eight-noded quadrilateral elements, leading to 13.689 unknowns (9.447 displacement unknowns and 4.242 traction unknowns). The FM-SGBEM analysis converges after 18 iterations. A comparison of the mean values $\bar{u}_{r}$ and $\bar{t}_{r}$ computed from the radial displacements and tractions at all nodes on the same surface or interface to the corresponding exact values of $u_{r}\left(r_{i}\right)$ and $t_{r}\left(r_{i}\right)$ (Table 3 ) shows solution errors that are consistent with the accuracy $\epsilon_{1}=10^{-3}$ chosen for GMRES.

\subsection{Bi-material fractured cylinder}

In this example, a simple bi-material cylinder (176 four-noded quadrilateral elements) containing a small internal crack is used for verifying the basic multi-domain formulation. Boundary conditions and materials properties are defined in Fig. 8a. The crack, located inside zone 2, is elliptical, with major semi-axis $a$ and minor semi-axis $b$; it is modelled using 48 quarter-point eight-noded quadrilateral elements. The crack size and position are chosen such that the COD $\phi$ is well approximated by the known analytical solution for the same crack buried in an infinite homogeneous medium. The mode-I component of the latter is given (see [42], Sec. 3.8) by

$$
\phi_{3}=\frac{2(1-\nu) \sigma_{33}^{0}}{\mu} \frac{b}{E(k)} \sqrt{1-\frac{x^{2}}{a^{2}}-\frac{y^{2}}{b^{2}}}
$$


(where $E(k)$ is the complete elliptic integral of the second kind and $k$ is defined by $k^{2}=1-b^{2} / a^{2}$ ). Numerical values of $\phi_{3}$ along the minor semi-axis are seen in Fig. 8b to agree well with the above analytical solution for several values of $b / a$.

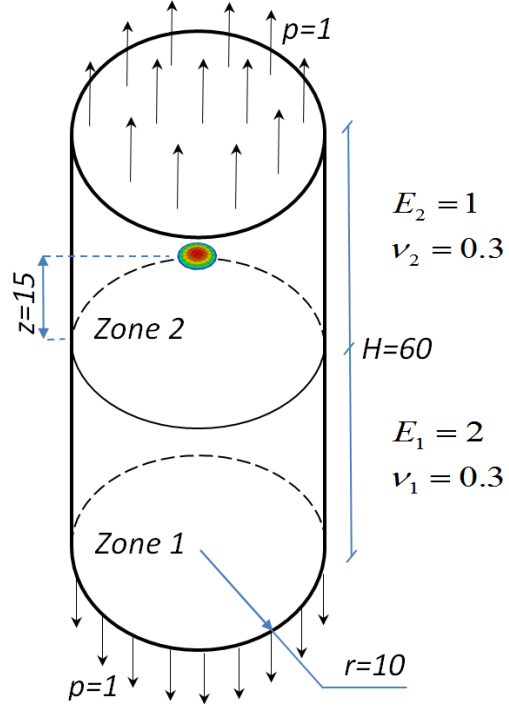

(a)

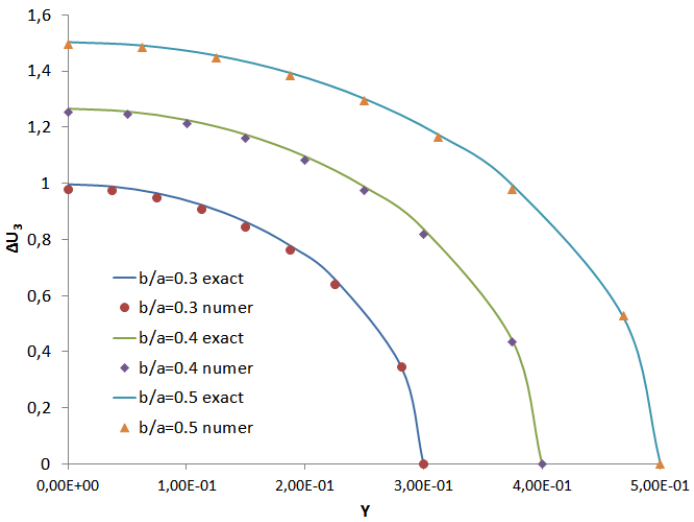

(b)

Figure 8: (a) Bi-material fractured cylinder (b) $\Delta u_{3}$ along the minor semi-axis of the elliptical crack, for several values of the aspect ratio $b / a$

\subsection{Bi-material cube with crack array}

We study now a bi-material cube occupying the region $0 \leq x_{1}, x_{2}, x_{3} \leq 100$, with the planar bimaterial interface defined by $x_{3}=50$. The cube is clamped on the bottom face $x_{3}=0$ and subjected to a uniform tensile load $p=1$ on the top face $x_{3}=100$ (Fig. 9a). The external boundary of the cube is modelled with 4.400 four-noded quadrilateral elements. The material properties are set to $E_{1}=1000, \nu_{1}=0,15$ (zone 1) and $E_{2}=2000, \nu_{2}=0.3$ (zone 2). The bimaterial cube contains many cracks, located on a cubic grid of step $d_{c}$ whose center coincides with the cube center. We consider three crack arrays $1,2,3$, respectively containing $n_{c}=10^{3}, 12^{3}, 14^{3}$ penny-shaped cracks of unit radius, with $d_{c}=10,8,6$. The truncation parameter $p$ in (13) is set to 7 . The maximal number of elements in a leaf is 30, the GMRES restart parameter is set to 50, and the stopping tolerance for F-GMRES is $\epsilon_{1}=10^{-3}$.

Table 4 shows computational data for FM-SGBEM analyses run on the three meshes. The CPU times per iteration grows linearly with the problem size $N$, consistently with the expected behavior

\begin{tabular}{|c||c|c|c|c|c|c|c|}
\hline Mesh & $N$ & $\ell$ & $\mathrm{M}$ & $\mathrm{CPU}(\mathrm{s})$, preparation & iters & $\mathrm{CPU}(\mathrm{s}) /$ iter & $\mathrm{CPU}(\mathrm{s})$, total \\
\hline 1 & 401,412 & 7 & 30 & 5,457 & 79 & 561 & 50,986 \\
2 & 683,148 & 7 & 30 & 12,197 & 66 & 1,204 & 95,584 \\
3 & $1,061,928$ & 7 & 30 & 11,903 & 102 & 1,872 & 206,114 \\
\hline
\end{tabular}

Table 4: Bi-material cube with crack array: computational data (with $\bar{\ell}$ and $M$ defined as in Table 2). 


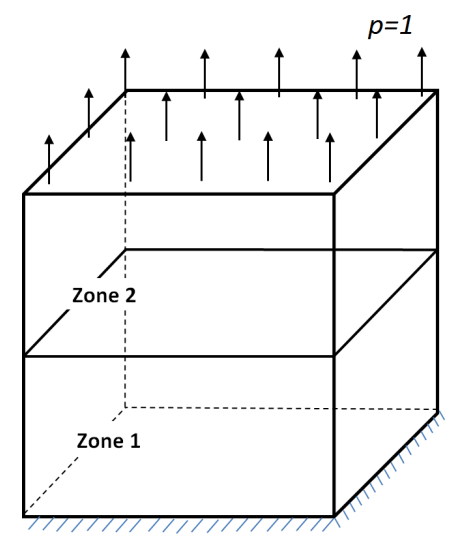

(a)

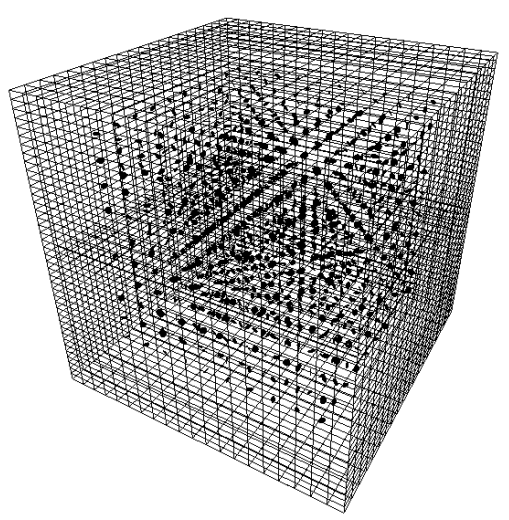

(b)

Figure 9: (a) Bi-material cube under uniform tensile load (b) Mesh 1: cube containing a 10x10x10 crack array

of the elastostatic FM-SGBEM algorithm. Nevertheless, one notes that the computation times for this example are significantly larger than those recorded for the unbounded single-zone problem of Sec. 3.3. This is because, by contrast with the unbounded fractured geometry involving only the bilinear form $\mathcal{B}_{\phi \phi}$, equation (29) for the present bi-material bounded configuration involves many other terms, which must all be evaluated. Furthermore, uneven spatial distribution of cracks may lead to leaf cells being located next to highly populated cells, increasing the work and memory required by near interactions.

\subsection{Cube with crack and inclusion arrays}

We now turn to the application of our method to composite materials made of inclusions embedded in a matrix. Configurations involving a cube of side $a$, subjected to a tensile load $p=1$ on its top face while clamped on its bottom face, and containing a system of $4 \times 4 \times 4$ spherical inclusions of radius $r_{i}$, are considered (Fig. 10). The inclusion centers are located on a regular cubic grid of step $d_{i}$. The matrix material characteristics are $E_{\text {solid }}=1, \nu_{\text {solid }}=0,3$, while the material parameters of the inclusions are randomly chosen such that $E_{\text {inclusion }}=1-10$, $\nu_{\text {inclusion }}=0,1-0,4$. In addition, the solid contains a system of $n_{c}^{3}$ internal penny-shaped cracks. These cracks are oriented randomly in space, while their centers are distributed on a regular cubic grid of step $d_{c}$. Each crack is either penny-shaped (with radius $\xi r_{c}$ ) or elliptical (with major semi-axis $\xi a_{c}$ and minor semi-axis $\xi b_{c}$ ), where $\xi \in[0.5,1]$ is a scaling coefficient chosen randomly in order to vary the size of each crack and inclusion. The geometrical parameters are set to $d_{i}=20, r_{i}=4$ for the inclusion array, $r_{c}=1, a_{c}=1, b_{c}=0.5$ for the crack array, and $a=80$ for the external cube size; moreover, four different crack arrays are considered, with $n_{c}=8,10,12,14$ and corresponding values of $d_{c}$ as given in Table 5. Each geometrical setting ensures that the cracks and inclusions are well-separated from each other and from the external boundary. The external surface of the cube and each inclusion boundary are made of 600 and 151 four-noded quadrilateral elements respectively. The crack is meshed with 48 eight-noded quadrilateral elements. The SGBEM equations (28) for each inclusion subdomain considered separately involve too few DOFs for the fast multipole treatment to be effective. Therefore, they are assembled in the traditional way, whereas the fast multipole treatment is of course applied to the SGBEM equations (28) relative to the cracked matrix subdomain, which involves the totality of the DOFs. 


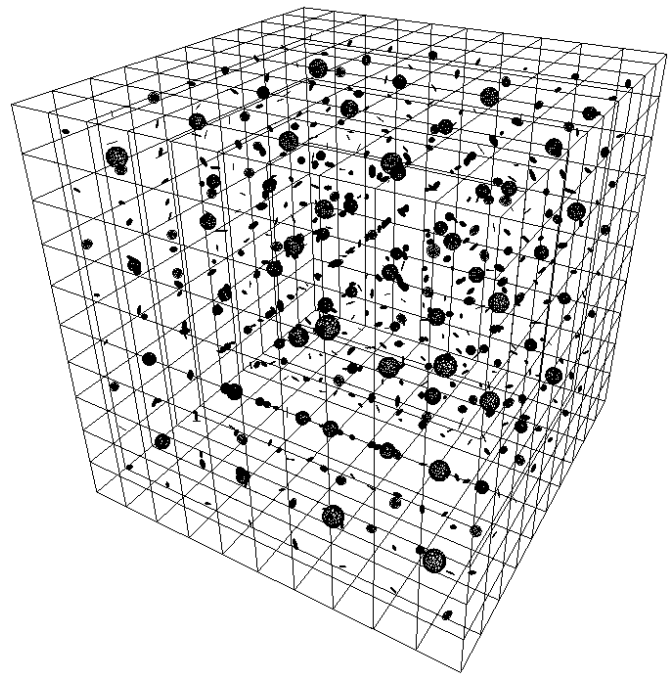

(a)

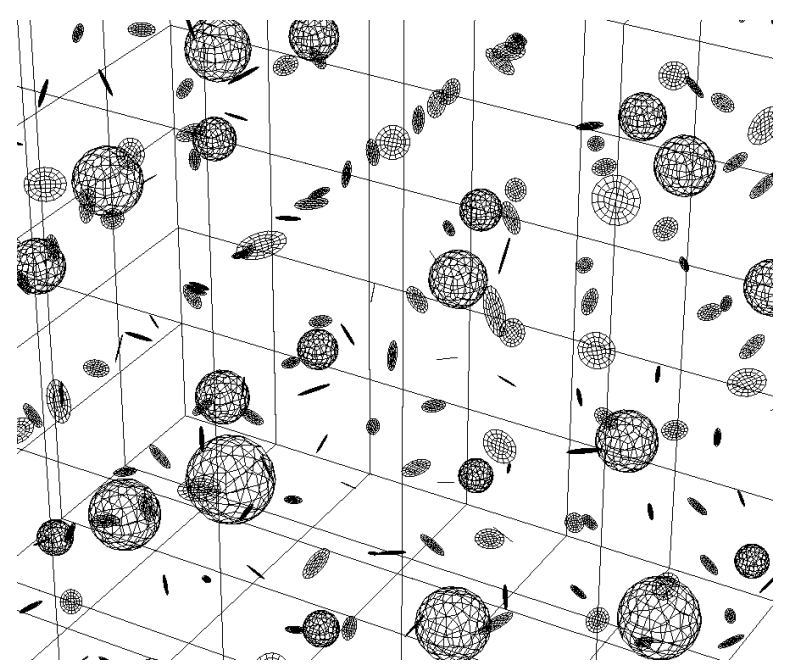

(b)

Figure 10: (a) Model of fractured composite material (4x4x4 spherical inclusions \& 8x8x8 cracks) (b) Interior view of cracks and inclusions

Table 5 shows computational data for FM-SGBEM analyses run on the four configurations. The CPU time per iteration is again seen to depend approximately linearly on the problem size $N$, while low outer iteration counts are achieved in all cases, for reasons similar to those given for the results of Table 2 .

\begin{tabular}{|c||c|c|c|c|c|c|c|c|c|}
\hline Mesh & $n_{c}$ & $d_{c}$ & $N$ & $M$ & $\bar{\ell}$ & CPU(s), preparation & iters & CPU(s)/iter & CPU(s), total \\
\hline 1 & 8 & 10 & 258,702 & 5 & 30 & 4,182 & 16 & 823 & 18,219 \\
2 & 10 & 7 & 447,558 & 8 & 30 & 6,165 & 15 & 1,274 & 26,832 \\
3 & 12 & 6 & 729,294 & 8 & 30 & 24,112 & 16 & 2,353 & 63,666 \\
4 & 14 & 5 & $1,122,468$ & 6 & 30 & 15,982 & 14 & 4,404 & 83,180 \\
\hline
\end{tabular}

Table 5: Cube with crack and inclusion arrays: computational data (with $\bar{\ell}$ and $M$ defined as in Table 2).

\section{Conclusions and outlook}

In this article, the formulation and implementation of a multi-level fast multipole SGBEM (FM-SGBEM) for piecewise-homogeneous elastic solid with cracks, applicable for arbitrary geometries and boundary conditions, was presented. Additional improvements of the FM-SGBEM performance were proposed and evaluated. Numerical results on test problems for BE models involving up to $3 \times 10^{6}$ unknowns were discussed. They show the applicability of the proposed FM-SGBEM to large-scale multi-zone and multi-crack configurations and in particular follow the predicted desirable trends of the elastostatic FM-SGBEM, such as a $O(N)$ complexity per iteration.

Future work includes extensions to more complex configurations involving surface-breaking or interface cracks, and the implementation of crack propagation algorithms. Practical modeling applications will address e.g. multi-layer road structures. 


\section{Acknowledgements}

This work is funded by the European Initiative Commission INTERREG IV - Upper Rhin Program (Project B20 TEM3)

\section{References}

\section{References}

[1] Bapat, M. S., Shen, L., Liu, Y. J. Adaptive fast multipole boundary element method for three-dimensional half-space acoustic wave problems. Eng. Anal. Bound. Elem., 33:1113-1123 (2009).

[2] Bebendorf, M. Hierarchical matrices: a means to efficiently solve elliptic boundary value problems, vol. 63 of Lecture Notes in Computational Science and Engineering. Springer (2008).

[3] Bonnet, M. Boundary integral equation methods for solids and fluids. John Wiley \& Sons (1999).

[4] Bonnet, M. Stability of crack fronts under Griffith criterion: a computational approach using integral equations and domain derivatives of potential energy. Comp. Meth. Appl. Mech. Eng., 173:337-364 (1999).

[5] Bonnet, M., Maier, G., Polizzotto, C. Symmetric Galerkin boundary element method. Appl. Mech. Rev., 51:669-704 (1998).

[6] Bui, H. D. An integral equation method for solving the problem of a plane crack of arbitary shape. J. Mech. Phys. Solids, 25:29-39 (1977).

[7] Chaillat, S., Bonnet, M., Semblat, J. F. A multi-level fast multipole BEM for 3-D elastodynamics in the frequency domain. Comp. Meth. Appl. Mech. Eng., 197:4233-4249 (2008).

[8] Chaillat, S., Semblat, J. F., Bonnet, M. A preconditioned 3-D multi-region fast multipole solver for seismic wave propagation in complex geometries. Commun. Comput. Phys. (to appear).

[9] Chen, T., Wang, B., Cen, Z., Wu, Z. A symmetric Galerkin multi-zone boundary element method for cohesive crack growth. Eng. Frac. Mech., 63:591-609 (1999).

[10] Chew, W. C. et al. Fast integral equation solvers in computational electromagnetics of complex structures. Eng. Anal. Bound. Elem., 27:803-823 (2003).

[11] Chow, E., Saad, Y. Experimental study of ILU preconditioners for indefinite matrices. J. Comp. Appl. Math., 86:387-414 (1997).

[12] Claeys, X., Hiptmair, R. Electromagnetic scattering at composite objects: a novel multi-trace boundary integral formulation. ESAIM: Math. Modell. Numer. Anal., 46:1421-1445 (2012).

[13] Costabel, M., Stephan, E. P. An improved boundary element Galerkin method for three-dimensional crack problems. Integ. Equ. Oper. Theory, 10:467-504 (1987).

[14] Darbas, M., Darrigrand, E., Lafranche, Y. Combining analytic preconditioner and Fast Multipole Method for the 3D Helmholtz equation. J. Comput. Phys., 236:289-316 (2013).

[15] Eshelby, J. D. The determination of the elastic field of an ellipsoidal inclusion and related problems. Proc. Roy. Soc. A, 241:376-396 (1957).

[16] Frangi, A., Di Gioia, A.. Multipole BEM for the evaluation of damping forces on MEMS. Comp. Mech., 37:24-31 (2005).

[17] Frangi, A., Novati, G., Springhetti, R., Rovizzi, M. 3D fracture analysis by the symmetric Galerkin BEM. Comp. Mech., 28:220-232 (2001).

[18] Ganguly, S., Layton, J. B., Balakrishna, C., Kane, J. H. A fully symmetric multi-zone Galerkin boundary element method. Int. J. Num. Meth. Eng., 44:991-1009 (1999).

[19] Gomez, J. E., Power, H. A multipole direct and indirect BEM for 2D cavity flow at low Reynolds number. Eng. Anal. Bound. Elem., 19:17-31 (1997).

[20] Gray, L. J., Chinta, B., Kane, J. H. Symmetric Galerkin fracture analysis. Eng. Anal. Bound. Elem., 15:103-109 (1995).

[21] Gray, L. J., Paulino, G. H. Symmetric Galerkin boundary integral formulation for interface and multi-zone problems. Int. J. Num. Meth. Eng., 40:3085-3101 (1997).

[22] Gray, L. J., Phan, A. V., Paulino, G. H., Kaplan, T. Improved quarter-point crack-tip element. Eng. Frac. Mech., 70:269-283 (2003).

[23] Greengard, L., Rokhlin, V. A fast algorithm for particle simulations. J. Comput. Phys., 73:325-348 (1987).

[24] Hackbusch, W., Nowak, Z. P. On the fast matrix multiplication in the boundary element method by panel clustering. Numer. Math., 54:463-491 (1989). 
[25] Hartmann, F. Introduction to boundary elements: theory and applications. Springer-Verlag (1989).

[26] Kallivokas, L., Juneja, T., Bielak, J. A symmetric Galerkin BEM variational framework for multi-domain interface problems. Comp. Meth. Appl. Mech. Eng., 194:3607-3636 (2005).

[27] Kupradze, V. D. (ed.). Three-dimensional problems of the mathematical theory of elasticity and thermoelasticity. North Holland (1979).

[28] Lai, Y.-S., Rodin, G. J. Fast boundary element method for three-dimensional solids comntaining many cracks. Eng. Anal. Bound. Elem., 27:845-852 (2003).

[29] Li, S., Mear, M. E., Xiao, L. Symmetric weak-form integral equation method for three-dimensional fracture analysis. Comp. Meth. Appl. Mech. Eng., 151:435-459 (1998).

[30] Liu, Y., Nishimura, N., Otani, Y., Takahashi, T., Chen, X. L., Munakata, H. A fast boundary element method for the analysis of fiber-reinforced composites based on a rigid-inclusion model. ASME J. Appl. Mech., 72:115-128 (2005).

[31] Margonari, M. Boundary element techniques for three dimensional problems in elastostatics. Ph.D. thesis, University of Trento (2004).

[32] Margonari, M., Bonnet, M. Fast multipole method applied to the coupling of elastostatic BEM with FEM. Comput. Struct., 83:700-717 (2005).

[33] Mura, T. Micromechanics of Defects in Solids. Martinus Nijhoff (1987).

[34] Nedelec, J. C. Integral equations with non integrable kernels. Integ. Equ. Oper. Theory, 5:562-572 (1982).

[35] Of, G., Steinbach, O., Wendland, W. L. The fast multipole method for the symmetric boundary integral formulation. IMA J. Num. Anal., 26:272-296 (2006).

[36] Pham, A. D., Mouhoubi, S., Bonnet, M., Chazallon, C. Fast multipole method applied to Symmetric Galerkin boundary element method for 3D elasticity and fracture problems. Eng. Anal. Bound. Elem., 36:1838-1847 (2012).

[37] Rizzo, F. J., Shippy, D. J., Rezayat, M. A boundary integral equation method for radiation and scattering of elastic waves in three dimensions. Int. J. Num. Meth. Eng., 21:115-129 (1985).

[38] Saad, Y. A flexible inner-outer preconditioned GMRES algorithm. SIAM J. Sci. Comput., 14:461-469 (1993).

[39] Saad, Y., Schultz, M.H. GMRES: a generalized minimal residual algorithm for solving nonsymmetric linear systems. SIAM J. Sci. Stat. Comput., 7:856-869 (1986).

[40] Sauter, S. A., Schwab, C. Boundary element methods. Springer-Verlag (2011).

[41] Sertel, K., Volakis, J. L. Incomplete LU preconditioner for FMM implementation. 26:265-267 (2000).

[42] Sneddon, I. N., Lowengrub, M. Crack problems in the classical theory of elasticity. Wiley (1969).

[43] Steinbach, O., Wendland, W. L. The construction of some efficient preconditioners in the boundary element method. Advances Comput. Math., 9:191-216 (1998).

[44] Sylvand, G. La méthode multipôle rapide en électromagnétisme : performances, parallélisation, applications. Ph.D. thesis, Ecole Nationale des Ponts et Chaussées, Noisy le Grand, France (2002).

[45] Takahashi, T., Nishimura, N., Kobayashi, S. A fast BIEM for three-dimensional elastodynamics in time domain. Eng. Anal. Bound. Elem., 28:165-180 (2004).

[46] Yasuda, Y., Sakuma, T. A technique for plane-symmetric sound field analysis in the fast multipole boundary element method. J. Comput. Acoust., 13:71-85 (2005).

[47] Yoshida, K.I. Applications of fast multipole method to boundary integral equation method. Ph.D. thesis, University of Kyoto (2001).

[48] Yoshida, S., Nishimura, N., Kobayashi, S. Application of fast multipole Galerkin boundary integral equation method to elastostatic crack problems in 3D. Int. J. Num. Meth. Eng., 50:525-547 (2001). 